\title{
In-Band Full-Duplex Wireless: Challenges and Opportunities
}

\author{
Ashutosh Sabharwal, Fellow, IEEE, Philip Schniter, Fellow, IEEE, Dongning Guo, Senior Member, IEEE, \\ Daniel W. Bliss, Senior Member, IEEE, Sampath Rangarajan, and Risto Wichman
}

(Invited Paper)

\begin{abstract}
In-band full-duplex (IBFD) operation has emerged as an attractive solution for increasing the throughput of wireless communication systems and networks. With IBFD, a wireless terminal is allowed to transmit and receive simultaneously in the same frequency band. This tutorial paper reviews the main concepts of IBFD wireless. One of the biggest practical impediments to IBFD operation is the presence of self-interference, i.e., the interference that the modem's transmitter causes to its own receiver. This tutorial surveys a wide range of IBFD self-interference mitigation techniques. Also discussed are numerous other research challenges and opportunities in the design and analysis of IBFD wireless systems.
\end{abstract}

Index Terms-Full-duplex, simultaneous transmit and receive, wireless communications.

\section{INTRODUCTION}

$\mathbf{T}$ HE wireless revolution has resulted in ever-increasing demands on our limited wireless spectrum, driving the quest for systems with higher spectral efficiency. Among the various ways to increase spectral efficiency, in-band full-duplex (IBFD) operation has recently gained attention. (See Section II-B for a rapidly growing list of publications.) The main idea behind inband full-duplex is as follows. Most contemporary communication systems contain terminals (e.g., base stations, relays, or mobiles) that function as both transmitters and receivers. Conventionally, these terminals operate in half-duplex or out-ofband full-duplex, meaning that they transmit and receive either at different times, or over different frequency bands. Enabling wireless terminals to transmit and receive simultaneously over the same frequency band (i.e., IBFD operation) offers the potential to double their spectral efficiency, as measured by the

Manuscript received November 26, 2013; revised March 27, 2014; accepted May 27, 2014. Date of publication June 12, 2014; date of current version October 3, 2014. The work of A. Sabharwal was supported in part by the National Science Foundation (NSF) under Grants CNS-1314822 and CNS1161596. The work of P. Schniter was supported in part by the United States Air Force under Air Force Contract FA8721-05-C-0002. The work of D. Guo was supported in part by NSF under Grant ECCS-1231828.

A. Sabharwal is with Rice University, Houston, TX 77005 USA (e-mail: ashu@rice.edu).

P. Schniter is with The Ohio State University, Columbus, OH 43210 USA.

D. Guo is with Northwestern University, Evanston, IL 60208 USA.

D. W. Bliss is with Arizona State University, Phoenix, AZ 85004 USA.

S. Rangarajan is with NEC Laboratories America, Inc., Princeton, NJ 08540 USA.

R. Wichman is with Aalto University, 02150 Espoo, Finland.

Color versions of one or more of the figures in this paper are available online at http://ieeexplore.ieee.org.

Digital Object Identifier 10.1109/JSAC.2014.2330193 number of information bits reliably communicated per second per $\mathrm{Hz}$, and thus is of great interest for next-generation wireless networks.

Beyond spectral efficiency, full-duplex concepts can also be advantageously used beyond the physical layer, such as at the access layer. From the access-layer point of view, enabling frame level in-band full-duplex, where a terminal is able to reliably receive an incoming frame while simultaneously transmitting an outgoing frame, could provide terminals with new capabilities. For example, terminals could detect collisions while transmitting in a contention-based network or receive instantaneous feedback from other terminals.

IBFD has, until now, not seen widespread use due to the potential debilitating effects of self-interference. Self-interference refers to the interference that a transmitting IBFD terminal causes to itself, which interferes with the desired signal being received by that terminal. To appreciate the impact of selfinterference, consider the following example in the context of contemporary femto-cell cellular systems [1]. Based on data provided in [1, Table 10-2], femto base stations and mobile handsets transmit at $21 \mathrm{dBm}$ with a receiver noise floor of $-100 \mathrm{dBm}$. If we assume $15 \mathrm{~dB}$ isolation ${ }^{1}$ between the base station's transmit and receive signal paths, then the base station's self-interference will be $21-15-(-100)=$ $106 \mathrm{~dB}$ above the noise floor. Thus, for a full-duplex basestation to achieve the link SNR equal to that of a half-duplex counterpart, it must suppress self-interference by more than $106 \mathrm{~dB}$ - a daunting amount. (See Fig. 1.) Moreover, the preceding analysis considers only femto-cell systems; systems with larger cells will require a higher transmit powers, and thus more self-interference suppression.

One might immediately wonder why an IBFD terminal cannot simply cancel self-interference by subtracting its transmitted signal from its received signal, given that the transmitted signal is known. To start, if one attempts this cancellation in the digital domain, i.e., after the receiver's analog-to-digital converter (ADC), then ADC dynamic range is a major bottleneck for the following reasons. ${ }^{2}$ Suppose that the IBFD

\footnotetext{
${ }^{1}$ Larger isolation is possible for different antenna architectures, e.g., see the experimental results reported in [2]-[6].

${ }^{2}$ In practice, limited ADC dynamic-range is one of many factors that limit performance. Others include oscillator phase-noise, nonlinearities associated with amplifiers and mixers, IQ mismatch (if direct conversion is employed), and channel estimation error.
} 


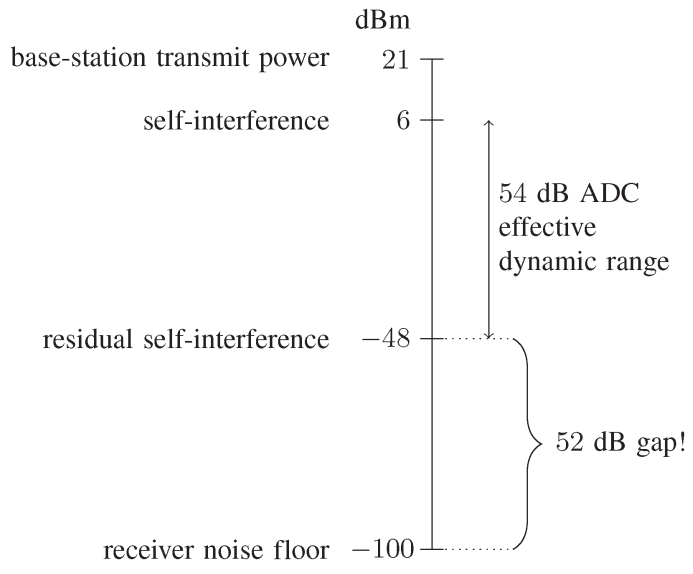

Fig. 1. Illustrative example of residual self-interference motivated by contemporary femto-cell cellular systems [1]. Even with perfect digital-domain selfinterference cancellation, the effects of limited ADC dynamic range cause a residual self-interference floor that is $52 \mathrm{~dB}$ above the desired receiver noise floor (i.e., the noise floor experienced by an otherwise equivalent half-duplex system).

terminal uses a $B$-bit ADC whose effective number of bits is ENOB. Although we may be tempted to use the 6.02 ENOB dB rule-of-thumb to compute the resulting quantization error floor, this rule-of-thumb holds only in the absence of clipping, for which we need to budget an additional headroom of $\approx 6 \mathrm{~dB}$ or 1 bit (depending on the received peak-to-average-powerratio). Moreover, to prevent the system from being quantization limited, it is typical to place the quantization-error floor $\approx 6 \mathrm{~dB}$ below the noise ${ }^{3}$ floor, which consumes 1 additional bit. Taking all of these factors into account, the effective dynamic range of an ADC is closer to 6.02(ENOB - 2) dB. (See the Appendix for a more precise analysis.) Thus, even if the self-interference is somehow perfectly cancelled in the digital domain, residual errors due to quantization and noise will remain at about $6.02(\mathrm{ENOB}-2) \mathrm{dB}$ below the level of the self-interference at the ADC input. So, if the IBFD terminal uses a 14-bit ADC with $\mathrm{ENOB}=11 \mathrm{bits}^{4}{ }^{4}$ thus yielding an effective dynamic range of $6.02(11-2) \approx 54 \mathrm{~dB}$, and if the self-interference power is $6 \mathrm{dBm}$ (as in the above femto-cell example), then a residual interference floor will remain at $6-54=-48 \mathrm{dBm}$, which is $-48-(-100)=52 \mathrm{~dB}$ above the desired femto-cell receivernoise-floor of $-100 \mathrm{dBm}$ (see Fig. 1).

From the above example, it is evident that digital-domain cancellation can suppress self-interference only up to the effective dynamic range of the $\mathrm{ADC}$, i.e., $\approx 6.02(\mathrm{ENOB}-2) \mathrm{dB}$. This limitation is a serious one, since industry trends over the last few decades suggest that commercial ADCs have improved significantly in sampling frequency but only marginally in ENOB, improving by only about 1 bit/decade [8] (see also [7] and [10]). We are thus strongly motivated to look for ways to reduce self-interference before the ADC. Scanning the litera-

\footnotetext{
${ }^{3}$ Here, "noise" refers to all other additive impairments beyond selfinterference and quantization error.

${ }^{4}$ We note that the difference between the actual and effective number of bits is ADC dependent and generally grows with the operating bandwidth. (See, for example, [7] and [8] for a more detailed discussion.) Our example is based on high-end Analog Devices ADCs, and specifically the 14-bit AD 9683 [9] designed for communications applications, which has an ENOB of $\approx 11$ bits over its designed range of sampling bandwidths.
}

ture on self-interference cancellation, we observe that the proposed techniques use a combination of wireless-propagationdomain techniques [2]-[6], [11]-[41], analog-circuit-domain techniques [2], [13], [24], [25], [28], [29], [31], [42]-[48] and/or digital-domain techniques [2], [3], [18]-[20], [23]-[25], [29], [31], [35], [36], [40], [41], [44], [49]-[53]. Moreover, each of these techniques may actively or passively suppress the selfinterference contributed by device-extrinsic scattering effects; see the detailed discussion in Section IV.

For example, in wireless-propagation-domain suppression, one aims to make the signal impinging upon the IBFD antennas free of self-interference. Typically this is accomplished by some combination of antenna directionality, cross-polarization, and transmit beamforming. In altering the transmit and/or receive antenna patterns, however, one must be careful to not also suppress the desired (outgoing and/or incoming) signal. This latter concern motivates analog-circuit-domain cancellation methods, which tap a copy of the transmitted signal from an appropriate location in the transmitter and subtract it (after proper gain, phase, and delay adjustment) from each receive antenna feed, since doing so leaves the (transmit and receive) propagation patterns uncompromised.

Although judicious application of the aforementioned techniques can yield impressive self-interference suppression in an anechoic chamber, performance can degrade considerably due to environmental effects, such as nearby reflections, that are impossible to predict at the time of system design. In essence, dealing with these device-extrinsic environmental effects requires learning and exploiting channel-state information (CSI), which is most easily handled in the digital domain. Once it is available, CSI can be exploited not only for selfinterference reduction, but also for resource allocation, e.g., the optimal allocation of limited signal power across space, time, and bandwidth [18], [40], [41], [54]-[57].

Why is IBFD important now? As evidenced by the recent literature discussed in Section II-B, there is significant interest in re-architecting terrestrial communications systems, such as WiFi and cellular systems, to leverage IBFD. A natural question is then, "Why now?," considering that some of the IBFD selfinterference mitigation techniques have been known for a while (see Section II). One driver is the widespread consensus that most "traditional" approaches to increasing spectral efficiency (e.g., advances in modulation, coding, MIMO) have by now been exhausted, leaving system designers willing to try more non-traditional approaches. But perhaps a bigger driver is the architectural progression towards short-range systems, such as small-cell systems and $\mathrm{WiFi}$, where the cell-edge path loss is less than that in traditional cellular systems, making the self-interference reduction problem much more manageable. This shift towards smaller cells, coupled with the fact that "consumer-faced" data networks dominate in prevalence and market-size over radars and repeaters (which have employed IBFD for a long time, as discussed in Section II), has sparked a renewed interest in IBFD.

Recently, several national, academic and corporate research laboratories [2]-[4], [18], [58] have taken the important first step of experimentally demonstrating the feasibility of IBFD in small-scale wireless communications environments (e.g., 
WiFi), thereby broadening the scope of IBFD beyond the traditional radar/repeater domain. Successfully applying IBFD to large-scale commercial wireless networks will, however, require wireless researchers to tackle many more challenges, since IBFD "removes" one of the basic constraints in the design of wireless networks, i.e., the half-duplex constraint. As a result, many aspects of network design and management will need to be reconsidered and redesigned. Even after these design challenges have been overcome, it still may be that not every node in the network is suitable for IBFD operation. For example, IBFD with small-form-factor devices still remains notoriously difficult and has, thus far, eluded successful experimental demonstration. In the case that sufficient selfinterference reduction is not possible, an alternative is "virtual full-duplex" signaling [59]-[61], which uses rapid on-off signaling to enable full-duplex at the frame level while using half-duplex at the physical layer, thereby achieving higher network throughput (see Section V-C). In summary, preliminary results have shown that IBFD has strong potential to increase the spectral efficiency of future wireless networks, although additional design innovations are essential before IBFD is used in operational networks.

The remainder of this article is structured as follows. In Section II, we review the history of IBFD wireless, including its longstanding use in radar systems. In Section III, we describe several opportunities for IBFD in current and future wireless communications systems. In Section IV, we detail a number of interference suppression techniques that enable IBFD, and in Section V, we outline important ongoing research topics in IBFD. Finally, in Section VI, we conclude.

\section{LiteratURE REVIEW}

In-band full-duplex wireless has a long history, and in fact the concept has been in use since at least the 1940s. In this section, we first discuss the history of IBFD in radar systems, and then we discuss the history of IBFD in wireless communication systems. Due to lack of space, we focus on providing an exhaustive (to the best of our knowledge) list of citations as a resource for researchers, rather than a detailed discussion of every citation.

\section{A. Full-Duplex Radars}

Continuous Wave (CW) radar systems use either two separate antennas (bistatic, as in Fig. 2(a)), or one shared antenna (mono-static, as in Fig. 2(b)), to transmit and receive simultaneously [62], in contrast to pulsed radar systems, which switch off the transmitter while radar returns are collected. Self-interference, often labeled as "transmitter leakage" in the radar literature [43], [50], is one of the key challenges faced in the design of all $\mathrm{CW}$ radars. The conventional $\mathrm{CW}$ radars of the 1940s and 1950s achieved isolation between the transmitter and receiver through antenna-separation-based path-loss in separate-antenna systems, or through the use of circulators in shared-antenna systems. (Circulators, illustrated in Fig. 2(b) and discussed further in Section IV-A, exploit nonlinear propagation in magnetic materials to isolate the incoming and outgoing signals [63].) Because only mild levels of isolation could be achieved using these techniques, keeping self-interference to a

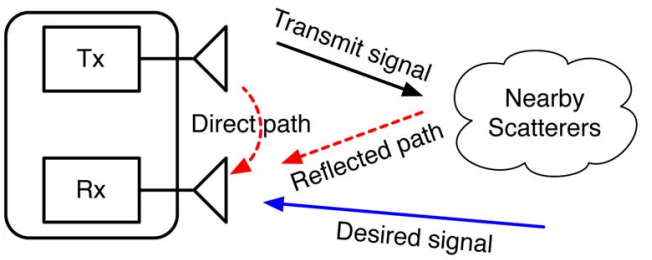

(a)

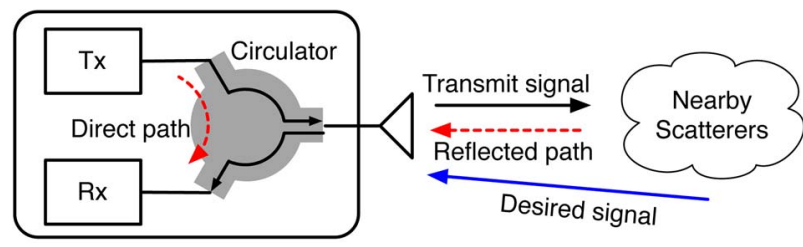

(b)

Fig. 2. Two methods of interfacing antennas to an IBFD wireless terminal. (a) Separate-antenna full-duplex. (b) Shared-antenna full-duplex.

manageable level required strongly limiting the transmit power, which then strongly limited the detectable range of targets. This restriction of $\mathrm{CW}$ radar to nearby targets (i.e., short ranges) turned out to be fortuitous, since detecting nearby targets with a pulsed radar system would require on/off switching times that are impractically small. Thus, the operational range of $\mathrm{CW}$ radars matched their need.

In the 1960s, the "feed-through nulling" approach, an analogcircuit-based form of self-interference cancellation, was proposed to increase the dynamic range of $\mathrm{CW}$ radars [42]. Although a total isolation of $60 \mathrm{~dB}$ was reported, the canceler required a $60+\mathrm{kg}$ block of precisely machined ferrite rotators and was very expensive. In 1990, an improved analog canceler was proposed in [11] that allowed adaptability to non-constant channel conditions, and its performance was demonstrated in [64]. Many refinements of the leakage canceler/isolator for monostatic CW radar have since been proposed [15]-[17], [45], [46], [48], [50]. It is worth noting that CW radar technology forms the basis of the now-ubiquitous Gen-2 RFID protocol [65]; see also the articles [47], [48] about ongoing research.

\section{B. Research Advances in In-Band Full-Duplex Wireless Communications}

While radar systems have extensively used IBFD, terrestrial wireless communication systems like cellular and WiFi have largely avoided IBFD, except in some special cases.

The first known application of IBFD to wireless communication came in the context of relaying, where in-band repeaters are used to increase coverage in a wireless communication system by receiving, amplifying, and re-transmitting the wireless signal in the same frequency band. (See Section III for more details on the relay topology.) Relays are often used in tunnels or difficult terrains, where laying wireline backhaul to each terminal is challenging and/or cost-prohibitive. For IBFD relays, the earliest known self-interference suppression techniques relied on increasing the physical separation between transmit and receive antennas (much like in bi-static $\mathrm{CW}$ radar) [14], [66]-[68]. Subsequently, a variety of circuit-domain selfinterference cancellation strategies (including analog and digital techniques as well as adaptive and non-adaptive ones) were proposed, e.g., [36], [44], [49], [51], and [69]-[71]. Further 
self-interference reductions were made possible through the use of antenna arrays, which allowed beamforming-based selfinterference nulling [18], [19], [21], [32]-[34], [36], [38], [41], [72]. Recently, a provision for IBFD relaying has been included in the 3GPP standard [73].

The information-theoretic limits of IBFD relaying have also been studied. Although early work [55], [74]-[77] focused on the ideal case of perfect self-interference cancellation, subsequent work [54], [72], [78]-[82] included the effects of residual self-interference. More recently, information-theoretic analyses of MIMO IBFD relaying that include the effects of channel estimation error, finite ADC resolution, and amplifier nonlinearities on residual self-interference have been considered in [41] and [83].

From the above body of work, it is clear that IBFD has a rich history in the context of wireless relays. For other wireless system configurations, although a few early papers do exist (e.g., [13]), the surge in interest has occurred only recently, after several academic/national/industrial research labs experimentally demonstrated the feasibility of bidirectional IBFD [2]-[4], [18], [25], [26], [29], [84], at least over short ranges. In bidirectional IBFD, two modems simultaneously exchange messages over the same frequency band, as further described in Section III. Much like CW radars, these implementations included both separate-antenna and shared-antenna architectures, as depicted in Fig. 2. However, due to the somewhat limited levels of self-interference suppression demonstrated in these initial experiments, they did not convincingly establish the feasibility of IBFD for practical WiFi and cellular systems. A second round of bidirectional IBFD experiments [24], [27], [28], [85], however, demonstrated improved self-interference suppression, to levels sufficient for WiFi networks. Further improvements were then experimentally demonstrated using advanced antenna designs in both separate-antenna [5], [6], [39] and shared-antenna [30], [31] architectures. Moreover, several patents covering IBFD have been awarded [58], [86], [87].

While the aforementioned literature proposed and experimentally demonstrated successful bidirectional IBFD designs, a different body of recent work [6], [88]-[93] has focused on characterizing the bottlenecks of practical IBFD architectures through a combination of theory and experiments, with the goal of improving future system implementations. Likewise, advanced self-interference cancellation solutions have been recently proposed [31], [40], [91], [94]-[96] that take into account the impairments introduced by the transmit radio chain. Moreover, information-theoretic analyses of MIMO bidirectional IBFD have been performed [40] that include the effects of channel estimation error, finite ADC resolution, and amplifier nonlinearities on residual self-interference. Even in out-ofband full-duplex transceivers, leakage due to transmit-chain impairments can cause self-interference at the receiver when the transmit and receive frequency bands are closely spaced. As a result, co-siting of TDD and FDD base stations operating on adjacent frequencies is not considered commercially viable [97]. The effects of such leakage, and associated cancellation algorithms, have been studied in [98]-[101].

Beyond the relay and bidirectional topologies, progress has already been made towards understanding the capacity gains

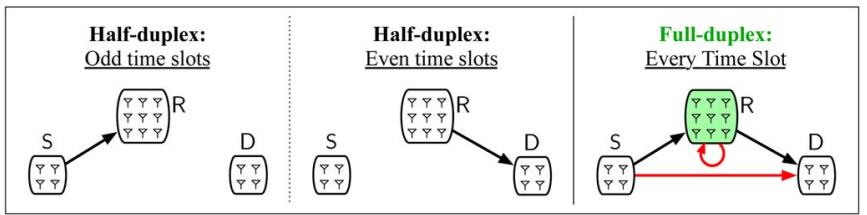

(a)

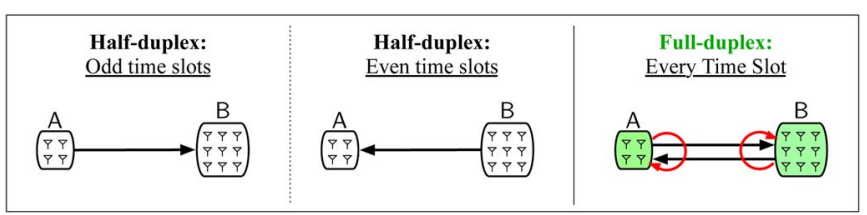

(b)

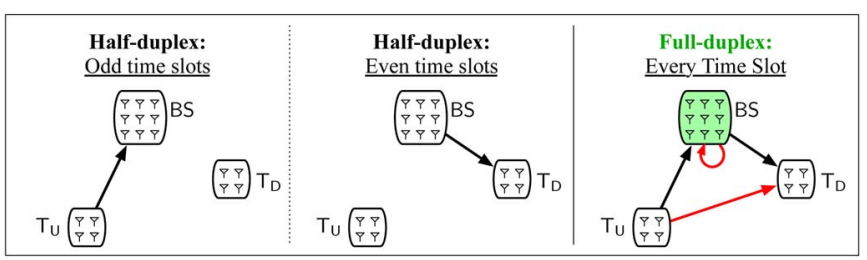

(c)

Fig. 3. Three example topologies that illustrate the implications of IBFD terminals at the network level. IBFD-capable terminals are shaded green, and IBFD-induced interference is shaded red. (a) Relay topology; (b) bidirectional topology; (c) base station topology.

from IBFD in more general multi-user network topologies, at least under perfect self-interference suppression [75], [102][104]. Similarly, distributed full-duplex [105], [106], and the application of full-duplex to cognitive radios [107] and multiband systems [108], [109], have also been considered.

Furthermore, progress has also been made towards understanding the impacts of full-duplex beyond the physical layer, such as in full-duplex medium-access protocols [110], [111] and in cross-layer optimization for full-duplex network scheduling [112]-[115]. Moreover, the advantages of fullduplex in neighbor discovery [60], mutual broadcasting (e.g., for the exchange of network state information) [61], and ranging/localization [116] have also been considered.

Based on the brief discussion above, it is clear that there exist many aspects of physical-layer IBFD and higher-layer full-duplex that require more thorough investigation. We will describe a few of the important open challenges in Section V.

\section{Opportunities to Leverage Full-Duplex}

To appreciate the gains from in-band full-duplex, it is instructive to look at three basic topologies in Fig. 3: the (a) relay topology, (b) bidirectional topology, and (c) base station topology. In each case, the terminals can have multiple antennas and the number of antennas per terminal can differ across terminals. The three basic topologies are inspired by both ad hoc and infrastructure-based (e.g., cellular and WiFi) networks. In an ad hoc network with IBFD terminals, different traffic patterns can result in all three basic topologies manifesting in different parts of the network. Similarly, the bidirectional and base station topologies are possible in infrastructure-based networks with IBFD terminals.

First consider the relay topology shown in Fig. 3(a), where terminal $\mathrm{R}$ acts as a relay for the single flow of data being sent from source terminal $S$ to destination terminal $D$. If terminal $R$ 
can only operate in half-duplex, ${ }^{5}$ then it would need to alternate between receiving from terminal $S$ and forwarding to terminal $D$, as shown in the left two panes of Fig. 3(a). However, if terminal $R$ can operate in IBFD, then it could receive and forward simultaneously (over the same frequency band), as shown on the right pane of Fig. 3(a). Thus, by operating in IBFD mode, the relay network can potentially double ${ }^{6}$ the spectral efficiency (measured in bits/second/Hz) relative to half-duplex operation. Note that the IBFD relay network requires only that the relay operate in full-duplex; neither the source nor the destination terminal needs to simultaneously transmit and receive.

Next consider the bidirectional topology shown in Fig. 3(b), where there are two data flows: terminal $A$ sends data to terminal $B$, and terminal $B$ sends data to terminal $A$. If either terminal $A$ or terminal $B$ only can operate in half-duplex, then communication from $A \rightarrow B$ cannot occur simultaneously with communication from $B \rightarrow A$, and the two communications must be performed over orthogonal time slots, as shown in the left two panes of Fig. 3(b). However, if terminal A and terminal $B$ both support IBFD operation, then communication from $A \rightarrow B$ can occur simultaneously with communication from $B \rightarrow A$, as shown in the right pane of Fig. 3(b), thereby potentially doubling ${ }^{7}$ the spectral efficiency relative to halfduplex.

Finally, consider the base station topology of Fig. 3(c), where there also exist two data flows: terminal $T_{U}$ sends data on the uplink to base station terminal BS, and terminal BS sends independent data on the downlink to terminal $T_{D}$. If terminal BS can only operate in half-duplex, then it would need to alternate between receiving from terminal $T_{U}$ in one time slot and transmitting to terminal $T_{D}$ in an orthogonal time slot, as shown in Fig. 3(c). However, if terminal BS could operate in IBFD, then it could support simultaneous in-band uplink and downlink communication, potentially doubling ${ }^{8}$ the spectral efficiency. Like in the relay topology, only terminal BS needs to support IBFD; terminals $T_{U}$ and $T_{D}$ need not simultaneously transmit and receive.

In Fig. 3, the black arrows represent desired-signal propagation and the red arrows depict interference, the latter of which

\footnotetext{
${ }^{5}$ Here and in the other topologies, similar statements about spectral efficiency could be made if "half-duplex" was replaced by "out-of-band full-duplex," and "orthogonal time-slot" was replaced by "orthogonal frequency band."

${ }^{6}$ Whether IBFD truly doubles the spectral efficiency of half-duplex or not depends on the power-constraint assumed for the comparison. Under a peak power constraint, the achievable spectral-efficiency of IBFD is exactly twice that of half-duplex (at all SNR). But under an average power constraint, the IBFD system could use only half the power used by IBFD during its transmission slot, resulting in an achievable spectral-efficiency gain of strictly less than 2 for any finite SNR [40], [41]. For example, if the SISO AWGN-channel capacity of the half-duplex system was $\log _{2}(1+\mathrm{SNR})$ then, under equal average power and perfect self-interference suppression, the corresponding capacity for IBFD would be $2 \log _{2}(1+\mathrm{SNR} / 2)$, yielding a spectral-efficiency gain of $2 \log _{2}(1+\mathrm{SNR} / 2) / \log _{2}(1+\mathrm{SNR})$, which increases monotonically from $1 \rightarrow 2$ as the SNR increases from $0 \rightarrow \infty$. This behavior becomes intuitive after recalling that communication systems transition from being power-limited to bandwidth-limited as SNR increases from $0 \rightarrow \infty$ and that power-limited systems benefit little from an increase in bandwidth [117], which is effectively what IBFD provides.

${ }^{7}$ The comments on the doubling of spectral efficiency from half-duplex to full-duplex in footnote 6 hold for arbitrary topologies.

${ }^{8}$ The comments on the doubling of spectral efficiency from half-duplex to full-duplex in footnote 6 hold for arbitrary topologies.
}

can come in two flavors. One is self-interference, which occurs when the signal transmitted by an IBFD terminal interferes with the reception of the desired incoming signal at the same terminal. The second is inter-terminal interference, which occurs in IBFD networks between terminals that may themselves be non-IBFD. For example, in the relay topology of Fig. 3(a), terminal S's transmission may cause interference to terminal D's reception, while in the base station topology of Fig. 3(c), terminal $T_{U}$ 's transmission may cause interference to terminal $T_{D}$ 's reception; such inter-terminal interference does not arise in the half-duplex versions of these networks. Achieving the ideal doubling of spectral efficiency in an IBFD network requires managing both self- and inter-terminal interference. Since selfinterference is the limiting factor of practical IBFD networks (e.g., it is much much stronger than inter-terminal interference), we will focus our attention on self-interference.

Finally, we note that generalizations of the three example topologies presented in Fig. 3 can be easily imagined. For example, the relay topology could support two simultaneous dataflows, one from $S \rightarrow D$ and another from $D \rightarrow S$, if terminals $S$, $\mathrm{R}$, and D all supported IBFD. Similarly, the three-terminal base station topology could support four simultaneous flows: $T_{U} \rightarrow$ $B S, B S \rightarrow T_{U}, T_{D} \rightarrow B S$, and $B S \rightarrow T_{D}$, if terminals $T_{U}, B S$, and $T_{D}$ all supported IBFD. Or, the base station topology could be extended beyond three terminals to a network with a single IBFD base station and $N>2$ mobiles, supporting up to $2 N$ simultaneous flows (if all terminals supported IBFD). Finally, the bidirectional topology could be extended to a network with $N>2$ IBFD terminals that supports up to $N(N-1)$ simultaneous data flows.

From the higher-layer perspective, the existence of IBFDcapable terminals removes one of the most fundamental constraints assumed in conventional protocol design: the (frame level) half-duplex constraint, and therefore expands the design space. This expanded design space offers new opportunities in protocol design that have the potential to increase network throughput. For instance, many existing network protocols require knowledge of the state of communicating terminals, such as their power, modulation format, code rate, ACK, and queue length (to name a few). With IBFD capability, these terminals could potentially exchange state information even while in the presence of other co-located simultaneous transmissions. The ability to multiplex control information alongside data transmission offers the possibility to reduce protocol overhead ${ }^{9}$ and significantly improve access-layer throughput.

\section{Techniques for SElf-Interference Reduction}

We now discuss several approaches to self-interference reduction for IBFD terminals. We find it convenient to partition them into three classes: propagation-domain, analog-circuitdomain, and digital-domain approaches. Another important distinction is whether they actively or passively mitigate the self-interference caused by device-extrinsic scattering.

\footnotetext{
${ }^{9}$ As a concrete example, access-layer protocol overhead in IEEE 802.11 has increased with each generation of standards, and exceeds 50\% for peak PHY rates in IEEE 802.11n [118]
} 


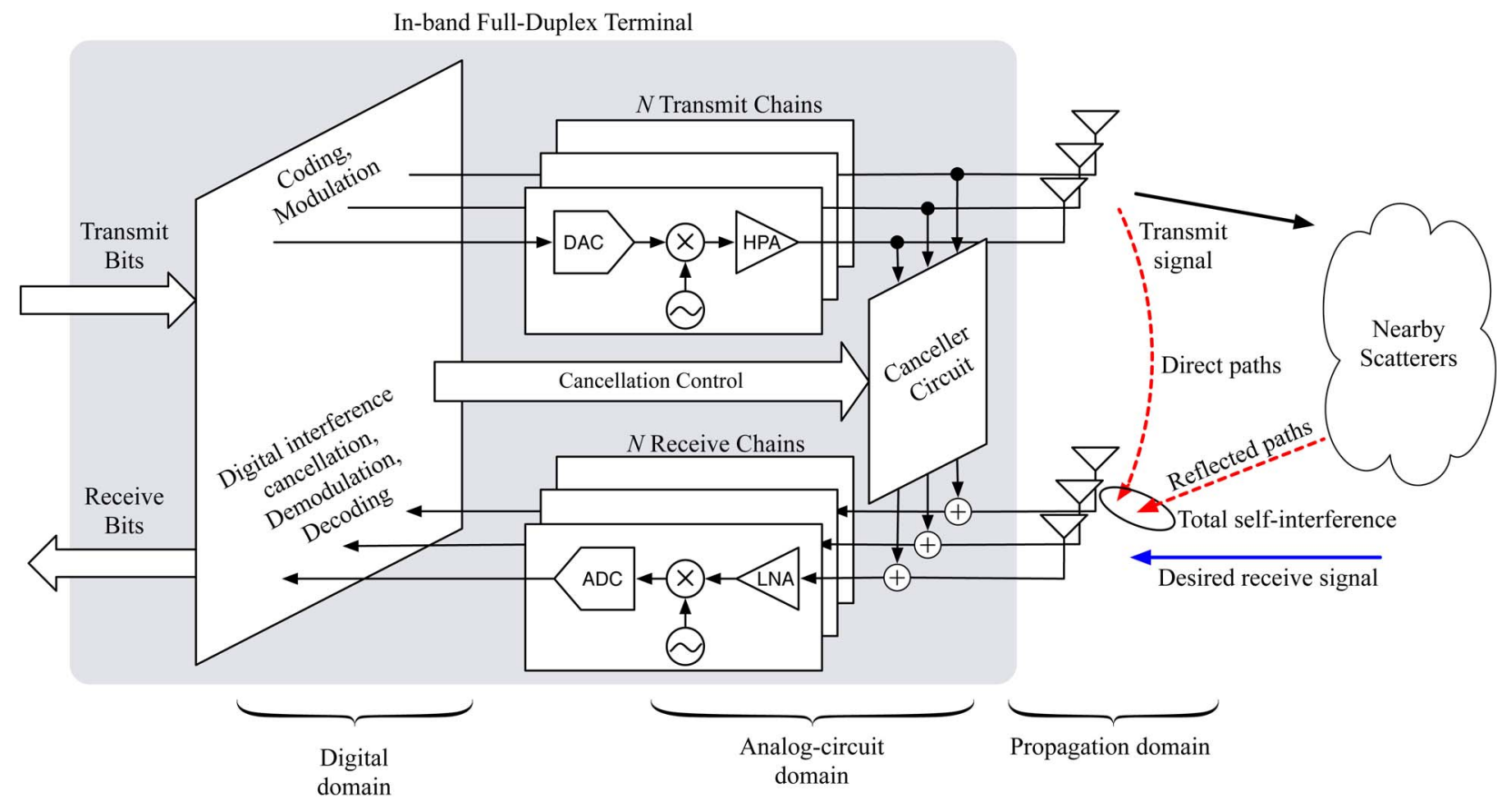

Fig. 4. Anatomy of a separate-antenna in-band full-duplex terminal with multiple transmit antennas and multiple receive antennas.

\section{A. Anatomy of an In-Band Full-Duplex Terminal}

To facilitate the discussion of self-interference suppression techniques, we first discuss the anatomy of a prototypical IBFD terminal and its immediate operating environment. Fig. 4 presents a schematic of a separate-antenna IBFD terminal with multiple transmit antennas and multiple receive antennas. A shared-antenna IBFD terminal would look similar, except that each transmit-receive antenna pair in Fig. 4 would be replaced by a circulator attached to a common antenna, as in Fig. 2(b). Differences between separate-antenna and shared-antenna systems will be further discussed in the sequel.

As can be seen from Fig. 4, the IBFD terminal accepts a transmit bitstream that is coded and modulated in the digital domain, producing a separate digital baseband signal for each transmit antenna. Each of these digital signals is then converted to analog with a digital-to-analog converter (DAC), upconverted to a high carrier frequency, amplified using a highpower amplifier (HPA), and radiated using a transmit antenna. In practice, this process will introduce several non-idealities into the transmit signal (e.g., DAC quantization noise, oscillator phase noise, and amplifier distortion), leading to small but important differences between the actual and intended transmit signals.

Simultaneously, and over the same frequency band, the IBFD terminal functions as a receiver. The signal picked up by each receive antenna is put through a separate hardware chain that includes a low-noise amplifier (LNA), downconverter, and ADC. The resulting digital baseband signals are then jointly processed in the digital domain (involving demodulation, interference cancellation, and bit decoding) to produce the received bitstream. Other aspects of the IBFD terminal, such as the canceler circuit visible in Fig. 4, as well as other options not visible from the diagram, will be discussed in the sequel.
There are two distinct ways in which the transmit and receive processing chains can be interfaced with antennas. One is the separate-antenna architecture, illustrated in both Fig. 2(a) and Fig. 4, and the other is the shared-antenna architecture, illustrated in Fig. 2(b). In the separate-antenna architecture, each transmit chain uses a dedicated radiating antenna and each receive chain uses a dedicated sensing antenna [2]-[6], [13], [24], [25], [27]-[29], [39], while in the shared-antenna architecture, each transmit-chain/receive-chain pair shares a common antenna [11], [15], [16], [30], [31]. Shared-antenna operation requires a shared-antenna duplexer that routes the transmit signal from the transmitter to the antenna and routes the signal received on the antenna to the receiver, all while isolating the receiver-chain from the transmit-chain. The most commonly used form of shared-antenna duplexer is the circulator, as illustrated in Fig. 2(b), which is a ferrite device that accomplishes the directional routing by exploiting non-linear propagation in magnetic materials [63]. Waveguide devices such as directional couplers [16] and coil-based devices such as hybrid transformers [119] can also be used to realize a sharedantenna duplexer. In the sequel, we use "circulator" when referring to any of these options. An IBFD terminal with $N$ transmit chains and $N$ receive chains will require $2 N$ antennas in a separate-antenna implementation or $N$ antennas plus $N$ circulators in a shared-antenna implementation.

As important as what happens inside the IBFD terminal is what happens just outside the terminal. We find it conceptually useful to decompose the received signal into three components: the desired received signal, the self-interference propagating directly from transmit chain to the receive chain, and the selfinterference reflecting off device-extrinsic scatters, especially nearby ones. In separate-antenna designs, the direct-path selfinterference comprises the signal propagating directly from the IBFD terminal's transmit antennas to its own receive antennas, 
as shown in Fig. 2(a), whereas, in shared-antenna designs, the direct-path self-interference is caused by circulator leakage (e.g., due to imperfect antenna matching), as shown in Fig. 2(b). While direct-path self-interference can be accurately characterized offline (e.g., in an anechoic chamber) and thus addressed at system design time, reflected-path self-interference cannot, since it depends on environmental effects that are changing and unpredictable. Thus, practical IBFD terminals must use a combination of techniques to suppress both direct-path and reflected-path self-interference while maintaining strong (incoming and outgoing) desired-signal gain.

\section{B. Propagation-Domain Self-Interference Suppression}

Wireless-propagation-domain isolation techniques aim to electromagnetically isolate the transmit chain from the receive chain, i.e., to suppress the self-interference before it manifests in the receive chain circuitry. The primary advantage to performing self-interference suppression in the propagation domain is that the downstream receiver hardware does not need to accurately process signals with a huge dynamic range. In separate-antenna systems, propagation-domain isolation is accomplished using a combination of path loss [2]-[4], [14], [27], [29], cross-polarization [6], [27], [39], [58], and antenna directionality [6], [39], while in shared-antenna systems it is accomplished using a circulator. ${ }^{10}$

In separate-antenna systems, the path loss between the IBFD terminal's transmit and receive antennas (or antenna arrays) can be increased by spacing them apart and/or by placing absorptive shielding between them, as quantified in [2], [6], [14], [29], [39]. Although path-loss-based techniques are attractive for reasons of simplicity, their effectiveness is greatly limited by the device form-factor: the smaller the device, the less room there is to implement such techniques. Cross-polarization offers an additional mechanism to electromagnetically isolate the IBFD transmit and receive antennas. For example, one may build an IBFD terminal that transmits only horizontally polarized signals and receives only vertically polarized signals with the goal of avoiding interference between them [6], [26], [27]. Similarly, with directional transmit and/or receive antennas (i.e., antennas with non-uniform radiation/sensing patterns), one may align their null directions in an attempt to achieve the same goal [5], [6]. In fact, one can build a highly directional transmit antenna from two omni-directional antennas using careful antenna placement [3], [4], [27], [58]: by placing a single receive antenna at precisely a location where the carrier waveforms are exactly 180 degrees out of phase, any narrowband signal modulated on those carriers will be near-perfectly canceled.

Although the preceding path-loss, cross-polarization, and antenna-directionality exploiting techniques are, in practice, somewhat hindered by issues like placement sensitivity and device integration, they can still be quite effective at suppressing direct-path self-interference. For example, using commercially available hardware, self-interference suppression levels of $74 \mathrm{~dB}$ have been attained in anechoic-chamber settings [6].

\footnotetext{
${ }^{10}$ In the case of the circulator, we consider the propagation domain to be the ferrite within the device.
}

Such anechoic figures are more representative of outdoor deployments than indoor ones, since the former are generally less reflective. In fact, when combined with analog cancellation (see Section IV-C) and digital cancellation (see Section IV-D), the design from [6] allowed near-perfect outdoor IBFD over ranges up to 150 meters. The Achilles heel of these techniques, however, is their sensitivity to reflected-path self-interference, whose channel characteristics are unknown at the time the system is designed. For example, the same design that delivered $74 \mathrm{~dB}$ suppression in the anechoic chamber delivered only $46 \mathrm{~dB}$ suppression in highly reflective indoor office environments [6]. Note that essentially the same problems occur in shared-antenna systems: a high-precision circulator (i.e., one with very low transmitter leakage) may suppress direct-path self-interference very well, but it has no way of discriminating between reflected-path self-interference and the desired receive-signal.

Handling device-extrinsic effects like nearby multipath scattering requires a channel aware method that can respond to these effects. Transmit beamforming is an example of a channel-aware propagation-domain self-interference suppression strategy, for which the IBFD terminal's multi-antenna transmit array is electronically steered (through the adjustment of per-antenna complex weights) in an attempt to zero the radiation pattern at each of the IBFD receive antennas. [18], [32]-[34], [36]-[38]. Of course, doing this successfully in the presence of reflected self-interference requires accurate knowledge of the (direct and reflected) self-interference channel gains and delays, which may be learned either explicitly via channelestimation or implicitly via weight adaptation. Moreover, each null placement consumes one degree-of-freedom in the weightvector design (beyond the constraint imposed by the transmit power constraint), and so effective transmit beamforming requires more IBFD transmit antennas than receive antennas. Finally, it may be worth noting that, while receive beamforming can also be performed, we would not classify it as propagationdomain interference suppression because the nulling would occur at the antenna-combining point, which resides in the circuit-domain.

Whether channel-aware or channel-unaware, the aforementioned propagation-domain self-interference suppression techniques have a potentially serious weakness: approaches that adjust the IBFD transmit and/or receive patterns to suppress self-interference might accidentally suppress the (incoming or outgoing) desired signals as well. This concern motivates the methods discussed in the sequel.

\section{Analog-Circuit-Domain Self-interference Cancellation}

Analog-circuit-domain cancellation techniques aim to suppress self-interference in the analog receive-chain circuitry, before the ADC. This suppression may occur either before or after the downconverter and the LNA. Fig. 4 shows one such configuration, where the transmit signal is tapped at the transmit antenna feed, electronically processed in the analog-circuit domain, and subtracted from the receive-antenna feed in order to cancel self-interference. But other options exist, such as tapping the transmit signal in the digital domain, applying 
the necessary gain/phase/delay adjustments digitally (where it is much easier to do so), and then converting ${ }^{11}$ it to the analog-circuit domain for use in self-interference cancellation [2], [24], [28], as depicted by the "cancellation control" path in Fig. 4. That said, tapping the outgoing signal as close as possible to the transmit antenna has advantages, since doing so better captures the presence of transmitter non-idealities like oscillator phase-noise and HPA distortion. Likewise, placing the cancellation point as close as possible to the receive antenna has the advantage of freeing more downstream hardware from the need to faithfully process signals with high dynamic range.

Like their propagation-domain counterparts, analog-circuitdomain cancellation techniques can either be channel-aware or channel-unaware. Channel-unaware techniques aim to cancel only the direct-path interference, whereas channel-aware techniques attempt to cancel both the direct- and reflectedpath interference. The "passive suppression" techniques [2], [5], [6], [24], [28] would be examples of channel-unaware analog-circuit-domain schemes, whereas the "active cancellation" techniques [2]-[4], [25], [31] would be examples of channel-aware analog-circuit-domain schemes.

For sufficiently narrowband signals, the (direct and/or reflected) self-interference channel can be well modeled as a complex gain and delay between every transmit and receive antenna pair. In this case, a separate-antenna IBFD terminal with single transmit and receive antennas would need to adjust only a single scalar complex cancellation gain and a single delay. A channel-unaware approach would do this adjustment once, when the system is designed/calibrated, while a channelaware design would continually adjust this gain and delay to compensate for changes in the reflection channel. Many implementations of the canceler circuit (as in Fig. 4) have been proposed; see, e.g., [2], [3], [27], and [31] for implementations that leverage a different mix of analog and digital processing components. The extension to multiple IBFD transmit and/or receive antennas will be straightforward, and will require tracking a separate gain and delay for every transmit-receive antenna pair.

For wideband signals, the direct-path self-interference can be mitigated using the same analog-circuit-domain techniques described above if the antenna gain and phase responses are engineered to be frequency-flat. The reflected-path self-interference channel, however, will in general be frequency-selective as a result of multipath propagation. This frequency selectivity makes analog-circuit-domain cancellation much more challenging, especially when the transmit signal is tapped and processed in the analog domain (as in Fig. 4), since then it would require adapting an analog filter for each transmit-receive antenna pair. Tapping the transmit signal in the digital domain, however, facilitates the use of digital adaptive filtering for reflected-path self-interference cancellation, which is typically much easier

\footnotetext{
${ }^{11}$ Converting to analog requires an additional DAC and upconverter (per transmit chain), but it does not require an additional high-power amplifier, which is one of the most power-hungry and expensive components in the transmit chain. We note that, while digital generation of the canceling signal can be implementationally more convenient, it does face imperfections due to DAC and up-converter phase noise, and thus this (as well as any) architectural choice needs to be carefully evaluated.
}

to implement, as proposed in [28] for wideband orthogonal frequency division multiplexing (OFDM) signals.

The preceding discussion demonstrates that there are many options and tradeoffs involved in the design of analog-circuitdomain interference cancellation. In summary, approaches that tap and cancel the interfering transmit signal very close to the antennas have the advantage of circumventing analog-domain non-idealities like HPA distortion and phase-noise, but the disadvantage of requiring analog-domain signal processing, which becomes especially difficult in the case of wideband reflectedpath self-interference. Meanwhile, approaches that tap and process the interfering transmit signal in the digital domain, for subsequent use in analog-domain cancellation, have the advantage of facilitating sophisticated adaptive digital signal processing techniques to reflected-path self-interference, but the disadvantage that their cancellation precision is limited by downstream analog-circuit non-idealities.

\section{Digital-Domain Self-Interference Cancellation}

Digital-domain cancellation techniques aim to cancel selfinterference after the ADC by applying sophisticated DSP techniques to the received signal. The advantage of working in the digital domain is that sophisticated processing becomes relatively easy. For example, while receive beamforming (where the per-antenna received signals are weighted by separate adaptive complex-valued gains before being summed together) could in principle be implemented in the analog-circuit domain, it is far more common in practice to implement it digitally [18], [19], [33], [38], for reasons of circuit complexity and power consumption. As described in Section I, however, the disadvantage to digital-domain cancellation is that the ADC's dynamicrange limits the amount of self-interference reduction that is possible. Thus, for digital-domain methods to be successful, a sufficient amount of the self-interference suppression must first occur before the ADC, using the propagation-domain and/or analog-circuit-domain methods described above. In this sense, we can think of digital-domain cancellation as the last line of defense against self-interference, where the goal is to cancel the self-interference left over from the propagation-domain and analog-circuit-domain approaches.

The first step to designing a digital-domain cancellation algorithm is to build a (baseband-equivalent) discrete-time system model that captures everything between the IBFD terminal's DAC and ADC, including the propagation-domain and analogcircuit-domain interference suppression techniques described earlier. An accurate model would need to capture not only the IBFD terminal itself, but also the inter-antenna propagation channels, and possibly even the other terminals in the IBFD network (which themselves may or may not support IBFD operation; recall Section III). Fig. 5 shows examples of such models for the relay and bidirectional topologies, taken from [40] and [41].

In Fig. 5, $\sqrt{\rho} \boldsymbol{H}$ and $\sqrt{\eta} \boldsymbol{H}$ are used to model signal and interference propagation, respectively, including the effects of propagation-domain and analog-circuit-domain suppression. In the frequency-selective (i.e., wideband signal) case with multiple transmit and receive antennas, these quantities would be 


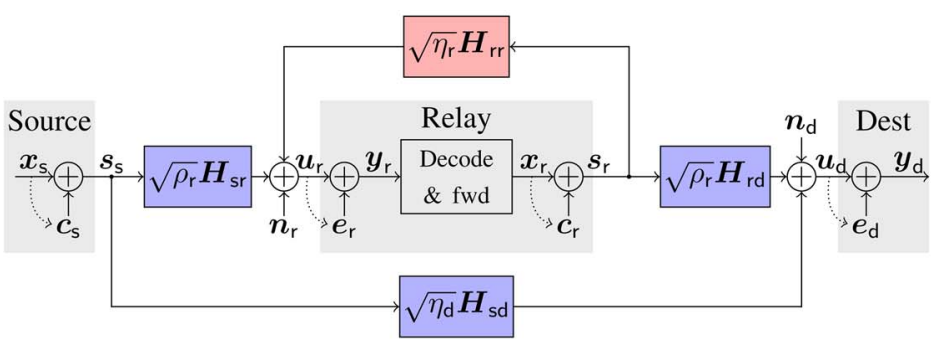

(a)

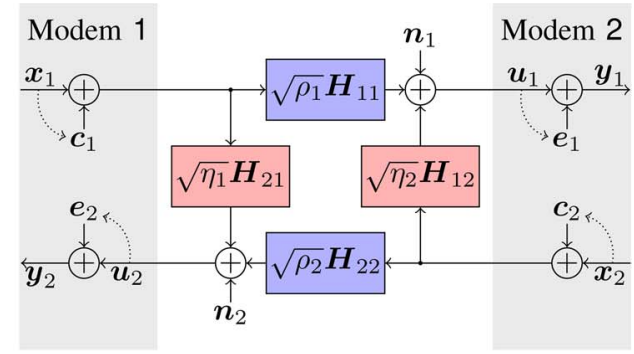

(b)

Fig. 5. Discrete-time baseband-equivalent system models for IBFD. The quantities $\sqrt{\rho} \boldsymbol{H}$ and $\sqrt{\eta} \boldsymbol{H}$ model signal and interference propagation, respectively, including the effects of propagation-domain and analog-circuit-domain suppression; $\boldsymbol{n}, \boldsymbol{c}$, and $\boldsymbol{e}$ represent channel noise, transmitter non-idealities, and receiver non-idealities, respectively; $\boldsymbol{x}$ and $\boldsymbol{s}$ represent intended and actual transmitted signals, respectively; and $\boldsymbol{u}$ and $\boldsymbol{y}$ represent the actual and ADC-measured received signals, respectively.

matrices of $z$-domain polynomials [52], [53], simplifying to matrices of complex numbers in the non-selective (i.e., narrowband signal) case, or scalar-valued entities in the case of single transmit and receive antennas. Fig. 5 also models the important difference between the intended and actual transmit signals, denoted by $\boldsymbol{x}$ and $s$, respectively, through the use of a "transmitter distortion" signal $\boldsymbol{c}$ that may depend (nonlinearly) on $x$. Recall that the intended and actual transmitted signals may differ as a result of DAC quantization noise [120], upconverter phase-noise, and HPA distortion. Similarly, Fig. 5 models the important difference between the received wireless signal and the corresponding ADC-output, denoted by $\boldsymbol{u}$ and $\boldsymbol{y}$, respectively, through the use of a "receiver distortion" signal $e$ that may depend (nonlinearly) on $\boldsymbol{u}$. Note that these transmitter and receiver distortions are independent of the additive channel noise $n$.

Focusing on the relay case in Fig. 5(a), a digital-domain cancellation scheme in the IBFD relay terminal might first compute an estimate $\sqrt{\eta}_{\mathrm{r}} \hat{\boldsymbol{H}}_{\mathrm{rr}}$ of the (partially-suppressed, directand reflected-path) self-interference channel and then use its knowledge of the intended transmit signal $\boldsymbol{x}_{\mathrm{r}}$ to form the selfinterference estimate $\sqrt{\eta}_{\mathrm{r}} \hat{\boldsymbol{H}}_{\mathrm{rr}} \boldsymbol{x}_{\mathrm{r}}$, which it could subtract from $\boldsymbol{y}_{\mathrm{r}}$ [36]. It is important to realize that, in doing so, the effects of channel estimation error, transmit distortion, and receive distortion remain. The non-linearity induced by the HPA can be modeled as a polynomial [121]. Thus, by estimating the parameters of the polynomial model used to construct the cancellation signal, some of the HPA non-linearities can be suppressed in the digital domain [31], [95], [122]. In multiple antenna systems, non-linear effects can also be suppressed with a combination of transmit and receive beam-forming [123]. By explicitly modeling and accounting for rest of the imperfections too, their deleterious effects on decoding and transmit beamforming can be possibly mitigated.

\section{Research Challenges AND Opportunities}

In this section, we discuss some of the key research challenges and opportunities that will help to enable the widespread application of IBFD to future wireless networks.

\section{A. Antenna and Circuit Design}

As is evident from the discussions in Section IV, a significant portion of IBFD self-interference cancellation relies on propagation-domain and analog-circuit-domain techniques. As wireless technology continues to spread, new types of mobile devices are being envisioned (e.g., wearable computing innovations like smart-watches, smart-glasses, health bands, and such), many of which have a very small form-factor. It is well known, even with half-duplex designs, that antennadesign bears most of the burden when special space- and shapeconstraints are imposed on mobile terminals. This underscores the importance of research on circumventing the impact of small-aperture antenna design on IBFD system performance.

While the advancement of digital circuitry has followed Moore's law, and thus benefited from exponential decreases in size and energy consumption, the advancement of analog circuitry has not. Over the last few decades, some RF processing tasks have successfully migrated to the digital domain (e.g., direct digital conversion) and thus have benefited from Moore's law, although others (e.g., power amplification) have not. Since the circuit-domain techniques discussed in Section IV-C often require additional (and often sophisticated) analog circuitry, IBFD terminals face the challenge of balancing self-interference cancellation performance with device cost, real-estate, and power consumption. For example, as shown in [88], [89], and [124], oscillator phase-noise directly impacts the performance of analog domain cancelers. Moreover, there exists a complex interplay between different components in the $\mathrm{RF}$ chain that affects the gains in spectral efficiency achievable through IBFD [91]. Thus, an important area of research is the design of low-power, low-cost, small-form-factor antennas, in addition to the analog and digital circuitry needed for self-interference suppression. In particular, additional gain may be achieved by judiciously designing small-form-factor antennas that facilitate subsequent analog self-interference cancellation.

\section{B. Physical-Layer Algorithm Design}

There are numerous important research opportunities for the advancement of IBFD physical-layer communications strategies, such as coding, modulation, power allocation, 
beamforming, channel estimation, equalization, digital interference cancellation, and decoding. We outline several below.

A necessary first step for the principled design of any digital communications system is an accurate statistical characterization of the effective channel seen by the system. In the case of IBFD, the effective channel, as seen in the digital domain, subsumes the analog circuitry and antennas as well as the propagation environment, and includes all of their non-idealities as well as the combined effects of any propagation-domain and/or analog-circuit-domain interference suppression strategies that may be employed, as well as ambient noise and external interference. Measurement-driven experimental studies [2], [24], [28], [85] have shown that, after self-interference has been (approximately) cancelled, the residual interference can often be well characterized by a Rician distribution whose K-factor is dependent on the amount of direct-path suppression. Furthermore, these studies have shown that the coherence interval and bandwidth of the residual self-interference depend on the local multipath environment and the terminal mobility, which are yet to be extensively characterized for IBFD terminals. Thus, an important area of research is the statistical characterization of the self-interference channel (with and without cancellation), especially for MIMO systems, where the characterization behaves as a function of array aperture, antenna element polarizations, inter-element distance, and element directionality. This statistical characterization will become the basis for not only system design but also information-theoretic performance analysis (see for example [125]).

Another line of research concerns optimal resource allocation, and in particular the optimal allocation of limited transmit power over space. The importance of spatial power allocation was already suggested in Section IV-B, where it was noted that alterations of the transmit-antenna directionality pattern for the purpose of self-interference reduction may also have the unintended consequence of reducing the power radiated on the intended receiver's antennas. The implication is that IBFD transmitters should be designed not to simply suppress self-interference but rather to optimize the balance between self-interference suppression and desired-signal radiation. Fortunately, with electronically steerable antenna arrays, it is possible to perform this optimization on-the-fly, in response to varying environmental conditions. Similar opportunities manifest when designing receive-beamforming weights (where array gain becomes the limited resource) or when jointly optimizing the IBFD terminals' transmit- and receive-beamforming weights. Moreover, when multiple terminals in an IBFD network use steerable antenna arrays, the spatial resource allocation problem extends to the network at large, since in that case multiple terminals can work together to spatially separate the desired and self-interference signals seen by each IBFD terminal. As a concrete example, the distant transmitter may be able to beamform in such a way that, when his signal impinges on the local IBFD terminal, it appears spatially orthogonal to the self-interference experienced by that terminal. Thus, an important area of research concerns the problem of formulating and solving spatial resource allocation problems in IBFD networks. There exists preliminary work on jointly optimizing transmit- and receive-arrays to maximize mutual information in bidirectional [41] and relay [40] MIMO IBFD networks under stylized channel models, but open problems remain in regards to more practical metrics and models.

While the above discussion focused on spatial resource allocation, the time and frequency domains present additional opportunities for optimization. Take, for example, the case where nearby fast-moving reflections cause the selfinterference power to remain extremely high even after all available self-interference reduction strategies have been implemented. In this case, forcing the IBFD terminal to operate in true IBFD mode may lead to a total loss of reliable communication. However, if each signaling epoch was split into two halves, each with an independently adjustable transmit power, the IBFD terminal could effectively fall into a timedomain half-duplex mode and communicate reliably with its partners, albeit at half-duplex rates [40], [41]. Then, when the nearby reflectors leave the scene, the same terminal could easily slip back into a true IBFD operating mode (via uniform temporal power-allocation) and thereby increase its spectral efficiency. Of course, a similar strategy could be implemented using two non-overlapping frequency bands or two orthogonal code subspaces. Moreover, in the wideband signal case, where the propagation channels are themselves frequency-selective, it can be seen that the resource allocation problem parameters change as a function of frequency. Thus, an important area of research concerns the problem of formulating and solving joint space-time-frequency resource allocation problems in IBFD networks.

Finally, it is important to study the fundamental performance limits of IBFD using metrics like Shannon capacity, outage capacity, diversity-multiplexing tradeoff, and network throughput, especially for MIMO systems. Since any such analysis will assume particular models for wireless signal propagation and analog/digital circuit non-idealities, the value of each given analysis will ultimately depend on the fidelity of its modeling assumptions. For bidirectional and relay topologies, bounds on the achievable-rate of MIMO IBFD were derived in [40], [41] under the standard isotropic Rayleigh-fading model for wireless signal propagation and a signal-power-dependent AWGN model for the analog/digital circuit non-idealities. Furthermore, since IBFD designs can be implemented with differing amounts of radio hardware resources (e.g., shared- versus separateantenna designs as discussed in Section II), capacity gains over half-duplex counterparts can be formulated by either equating RF-circuit resources or number-of-antennas [126], [127], leading to different conclusions under different use cases. Clearly, the consideration of other performance metrics, as well as more realistic propagation/circuit models, present important open areas of research.

\section{Network Foundations and Protocol Design}

A typical wireless terminal of today is but one drop of a sea of networked terminals. Different networks often have to share the same radio spectrum. To fully exploit the potentials of IBFD, careful engineering in medium-access control and higher-layer protocols is as important as that in the physical 
layer. Research results on the networking aspects of IBFD are emerging and there are abundant research opportunities in this direction. Perhaps the biggest research challenge lies in developing a foundation for network design where all or some of the terminals are capable of IBFD operation. We discuss several key issues in the following.

IBFD has the potential to significantly increase the overall throughput of a wireless network, beyond simply doubling the spectral efficiency of a point-to-point link. This is because IBFD removes a major scheduling constraint due to selfcollision, so that a terminal may transmit to a second or a group of terminals and simultaneously receive from a third or another group of terminals. As an example, consider a specific situation in a mobile $a d$ hoc network (MANET), where every terminal wishes to multicast the same data to all of its one-hop neighboring terminals. Without IBFD, at most one transmission can be successful in each frequency band in each neighborhood, because if multiple terminals simultaneously transmit in the same frequency band, they miss each other's transmission. IBFD enables all terminals to transmit at the same time, where each terminal receives a superposition of neighbors' transmissions for decoding. A preliminary study in [59] indicates the throughput gain over random access schemes (such as ALOHA) increases without bound as the number of neighbors increases. Fundamentally, with simultaneous transmissions, each receiver experiences an ergodic multiaccess channel (hence the sum energy is collected), whereas, with intermittent transmissions following a random access protocol, each receiver experiences a nonergodic channel at the frame level, where some transmissions are lost and energy is wasted due to collisions.

From the network perspective, IBFD also allows more concurrent transmissions to be packed in a given area. An interesting problem is to analyze the throughput of a network of randomly deployed terminals using stochastic geometry [128], [129]. IBFD may have implications on network layer protocols such as routing, as the routing algorithm may not need to try to avoid intersecting routes, which may reduce the length of the route and the overall interference. In addition, the system throughput may benefit by letting terminals en route jointly process bidirectional flows, perhaps also by leveraging network coding techniques [130]-[132].

Admittedly, the information capacity of a general wireless network, with or without IBFD, is widely open. Perhaps it would be simpler to characterize the capacity advantage due to IBFD in various network scenarios, which would provide useful guidelines to practical design.

Another interesting question concerns the implications of IBFD on practical WiFi-type networks. An IBFD terminal can be continuously cognitive, even during its own transmission, allowing the terminal to abort its transmission immediately upon collision. Equally important is improved throughput, e.g., by letting an access point transmit and receive packets simultaneously. IBFD medium access control schemes for WiFi were proposed in [29] and [25], with substantial gains reported. The former includes mechanisms to share random backoff counters and to snoop for full duplex transmission opportunities, whereas the latter greatly alleviates the hidden node problem.
IBFD may also lead to dramatically improved performance in other familiar network scenarios. In a cognitive radio network, secondary IBFD terminals may cause substantially smaller interference to primary users, and can sustain continuous transmission because it does not need to stop transmission in order to listen to the channel. Unlike a half-duplex relay, an IBFD relay can decouple the incoming link and the outgoing link to easy scheduling. It is also possible to relay the received signal instantaneously with little delay, or relay partially decoded packets before the entire packet is even received. IBFD also enables instantaneous feedback between a pair of communicating nodes. This allows the same transmission rate and higher reliability to be achieved by using shorter variablelength error control codes [133], [134], reducing the decoding delay and complexity. How to fully take advantage of IBFD in such network scenarios is an interesting problem.

Whether IBFD should be transparent to higher layers (MAC layer and above) or be exploited is an important question. Transparency ensures that protocols designed for half-duplex radios can be used without any changes. The answer resides in size of the gain achieved by novel protocols taking full advantage of IBFD.

To fully exploit the multiaccess nature of the wireless medium in conjunction with IBFD, one has to overcome the challenge of decoding in presence of co-channel interference or jointly decoding multiple users. There is a large body of literature on multiuser detection (see, e.g., [135], [136] and references therein). Compressed sensing has proven to be a useful technique for multiuser decoding in certain applications [60], [61]. Whether multiple transmissions can and should be synchronized or not is an important issue in practical design.

It is important to note that many of the advantages of full duplexing in a wireless network are retained as long as the terminals are full-duplex at the frame level, even if they are still half-duplex at the symbol level. In particular, when the power difference between the transmit and the receive signals becomes too large, it may be infeasible or too expensive to cancel selfinterference. A special signaling technique was proposed in [59] to achieve virtual IBFD. The idea is to introduce off-slots (in time, frequency, or both) within each frame of a terminal's transmissions, through which the terminal can collect useful signals without contamination by self-interference. This allows a frame to be received (albeit with slot erasures) simultaneously as another frame is being transmitted. The technique has found applications in neighbor discovery [60], mutual broadcasting (e.g., for exchanging link and network state information) [61] as well as ranging and localization [116]).

\section{CONCLUSION}

In-band full-duplex offers numerous opportunities for increasing the spectral efficiency of wireless networks. The opportunities are understandably accompanied with a number of challenges at all layers, ranging from antenna and circuit design, to the development of theoretical foundations for wireless networks with IBFD terminals. Much work remains to be done, and an inter-disciplinary approach will be essential to meet the numerous challenges ahead. In short, these are 
exciting times for wireless researchers and, in turn, the mobile telecommunications industry, who can look forward to more efficient future-generation wireless networks.

\section{APPENDIX}

This appendix shows that the effective dynamic range of the ADC is approximately $6.02(\mathrm{ENOB}-2) \mathrm{dB}$ when the system is designed so that the $\mathrm{ADC}$ quantization error is $6.02 \mathrm{~dB}$ (i.e., 1 bit) below the noise power and the signal's peak-to-averagepower ratio (PAPR) is $5 \mathrm{~dB} .{ }^{12}$

Suppose that the signal coming into the ADC is $x=d+s+$ $n$, where $d$ is the desired signal, $s$ is the self-interference, and $n$ is the "noise," which collects all perturbations beyond selfinterference and quantization error. Then the ADC output will be $y=x+e$ where, for an ADC with an effective number of bits of ENOB, the quantization error $e$ will have variance

$$
\sigma_{e}^{2}=\mathrm{PAPR}_{x} \sigma_{x}^{2} 10^{-6.02 \mathrm{ENOB} / 10}
$$

Given that each additional bit of ENOB halves the amplitude of the quantization error, we note that the multiplier $-6.02=$ $20 \log _{10}(1 / 2)$ above plays the role of converting from bits to $\mathrm{dB}$. Likewise, the multiplier $\mathrm{PAPR}_{x}$ above is used to prevent clipping. In particular, $\mathrm{PAPR}_{x}$ denotes the peak-to-average power level of $x$, defined such that $\sqrt{\mathrm{PAPR}_{x} \sigma_{x}^{2}}$ is the maximum voltage level at the input to the ADC.

The signal resulting from perfect digital-domain selfinterference cancellation, i.e., $z \triangleq y-s=d+n+e$, will have a signal-to-interference-plus-noise ratio (SINR) of $\sigma_{d}^{2} /\left(\sigma_{n}^{2}+\sigma_{e}^{2}\right)$ if we treat $e$ as uncorrelated with $n$. Assuming that the ENOB has been chosen so that $\sigma_{e}^{2}=\sigma_{n}^{2} / 4$ (i.e., the quantization error power is $6.02 \mathrm{~dB}$ or 1 bit below the noise power), then the SINR would be

$$
\begin{aligned}
\mathrm{SINR} & =\frac{\sigma_{d}^{2}}{\sigma_{e}^{2}+\sigma_{n}^{2}} \\
& =\frac{\sigma_{d}^{2}}{5 \sigma_{e}^{2}} \\
& =\frac{\sigma_{d}^{2}}{5 \operatorname{PAPR}_{x}\left(\sigma_{d}^{2}+\sigma_{s}^{2}+\sigma_{n}^{2}\right) 10^{-6.02 \mathrm{ENOB} / 10}},
\end{aligned}
$$

where $d, s$, and $n$ are assumed uncorrelated (so that $\sigma_{x}^{2}=\sigma_{d}^{2}+$ $\sigma_{s}^{2}+\sigma_{n}^{2}$ ). We can then upper-bound the SINR by

$$
\overline{\operatorname{SINR}}=\frac{\sigma_{d}^{2}}{5 \operatorname{PAPR}_{x} \sigma_{s}^{2} 10^{-6.02 \mathrm{ENOB} / 10}}
$$

noting that the bound will be tight when $\sigma_{s}^{2} \gg \sigma_{d}^{2}+\sigma_{n}^{2}$ (i.e., when the self-interference dominates), which is precisely the

\footnotetext{
${ }^{12}$ The peak-to-average power level varies with signal type, and in particular with symbol constellation, the oversampling factor above the baud rate, the number of carriers (in a multi-carrier system), and the number of antennas (in a MIMO) system [137]. For HDSPA waveforms, an often-cited Motorola technical report [138] predicts PAPR between 3 and $5.9 \mathrm{~dB}$, while other test models [139] estimate a PAPR of $\approx 5.7$.
}

case of interest. Writing the previous expression in $\mathrm{dB}$, we have

$$
\left.\overline{\operatorname{SINR}}\right|_{\mathrm{dB}}=\left.\frac{\sigma_{d}^{2}}{\sigma_{s}^{2}}\right|_{\mathrm{dB}}+6.02 \mathrm{ENOB}-\left.\mathrm{PAPR}_{x}\right|_{\mathrm{dB}}-7.0 .
$$

The above expression shows that, after perfect digital-domain self-interference cancellation, the SINR (in dB) is tightly upper bounded by the pre-ADC signal-to-interference ratio (i.e., $\sigma_{d}^{2} / \sigma_{s}^{2}$ in $\mathrm{dB}$ ) plus the dynamic range of the $\mathrm{ADC}$ (i.e., 6.02ENOB dB) plus a penalty factor of $-\left.\mathrm{PAPR}_{x}\right|_{\mathrm{dB}}-7 \mathrm{~dB}$. If we use ${ }^{13} \operatorname{PAPR}_{x}=5 \mathrm{~dB}$, then the penalty factor becomes $-12 \mathrm{~dB}$, which amounts to a loss of 2 bits relative to the ENOB.

\section{ACKNOWLEDGMENT}

The authors would like to thank the former Editor-in-Chief L. B. Milstein for suggesting to write this review article as a vehicle to introduce in-band full-duplex concepts to the larger research audience. The authors would like to thank Evan Everett, Achaleshwar Sahai, and Jingwen Bai at Rice University for their generous help in preparing the article, which included discussions of prior work, the development of artwork in this paper, and valuable feedback on many drafts.

\section{REFERENCES}

[1] "Interference Management in UMTS Femtocells," London, U.K., Feb. 2010.

[2] M. Duarte and A. Sabharwal, "Full-duplex wireless communications using off-the-shelf radios: Feasibility and first results," in Proc. Asilomar Conf. Signals, Syst. Comput., 2010, pp. 1558-1562.

[3] J. I. Choi, M. Jain, K. Srinivasan, P. Levis, and S. Katti, "Achieving single channel, full duplex wireless communications," in Proc. ACM MobiCom, 2010, pp. 1-12.

[4] M. A. Khojastepour, K. Sundaresan, S. Rangarajan, X. Zhang, and S. Barghi, "The case for antenna cancellation for scalable full-duplex wireless communications," in Proc. 10th ACM Workshop HotNets, 2011, p. 17.

[5] E. Everett, M. Duarte, C. Dick, and A. Sabharwal, "Empowering fullduplex wireless communication by exploiting directional diversity," in Proc. Asilomar Conf. Signals, Syst. Comput., 2011, pp. 2002-2006.

[6] E. Everett, A. Sahai, and A. Sabharwal, "Passive self-interference suppression for full-duplex infrastructure nodes," IEEE Trans. Wireless Commun., vol. 13, no. 2, pp. 680-694, Feb. 2014, to be published.

[7] R. H. Walden, "Analog-to-digital converter survey and analysis," IEEE J. Sel. Areas Commun., vol. 17, no. 4, pp. 539-550, 1999.

[8] P. Angeletti, G. Gallinaro, L. Hili, and X. Maufroid, "Evolution of analog to digital conversion technology for wideband space applications," in Proc. 23rd AIAA ICSSC, Rome, Italy, 2005, pp. 25-28.

[9] AD9683, Analog Devices, Norwood, MA, USA, 2014.

[10] J. Corcoran and K. Poulton, "Analog-to-digital converters-20 years of progress in "Agilent" oscilloscopes," Agilent Meas. J., vol. 1, pp. 34-40, Mar. 2007.

[11] P. Beasley, A. Stove, B. Reits, and B. As, "Solving the problems of a single antenna frequency modulated CW radar," in Proc. IEEE Int. Radar Conf., 1990, pp. 391-395.

[12] M. Cryan, P. Hall, S. Tsang, and J. Sha, "Integrated active antenna with full duplex operation," IEEE Trans. Microw. Theory Tech., vol. 45, no. 10, pp. 1742-1748, Oct. 1997.

[13] S. Chen, M. A. Beach, and J. P. McGeehan, "Division-free duplex for wireless applications," IEEE Electron. Lett., vol. 34, no. 2, pp. 147-148, Jan. 1998.

[14] C. Anderson et al., "Antenna isolation, wideband multipath propagation measurements, and interference mitigation for on-frequency repeaters," in Proc. IEEE SoutheastCon, Mar. 2004, pp. 110-114. 
[15] J. G. Kim, S. Ko, S. Jeon, J. W. Park, and S. Hong, "Balanced topology to cancel Tx leakage in CW radar," IEEE Microw. Wireless Compon. Lett., vol. 14 , no. 9 , pp. $443-445$, Sep. 2004.

[16] W.-K. Kim et al., "A passive circulator for RFID application with high isolation using a directional coupler," in Proc. 36th Eur. Microw. Conf., 2006, pp. 196-199, IEEE.

[17] C.-Y. Kim, J.-G. Kim, and S. Hong, "A quadrature radar topology with TX leakage canceller for 24-GHz radar applications," IEEE Trans. Microw. Theory Tech., vol. 55, no. 7, pp. 1438-1444, Jul. 2007.

[18] D. W. Bliss, P. A. Parker, and A. R. Margetts, "Simultaneous transmission and reception for improved wireless network performance," in Proc. IEEE Statist. Signal Process. Workshop, Aug. 2007, pp. 478-482.

[19] J. Sangiamwong, T. Asai, J. Hagiwara, Y. Okumura, and T. Ohya, "Joint multi-filter design for full-duplex MU-MIMO relaying," in Proc. IEEE VTC-Spring, 2009, pp. 1-5.

[20] T. Riihonen, S. Werner, and R. Wichman, "Spatial loop interference suppression in full-duplex MIMO relays," in Proc. Asilomar Conf. Signals, Syst. Comput., Nov. 2009, pp. 1508-1512.

[21] P. Larsson and M. Prytz, "MIMO on-frequency repeater with self-interference cancellation and mitigation," in Proc. IEEE 69th VTC-Spring, 2009, pp. 1-5.

[22] Y. Hua, "An overview of beamforming and power allocation for MIMO relays," in Proc. MILCOM, 2010, pp. 375-380.

[23] T. Riihonen, S. Werner, and R. Wichman, "Residual self-interference in full-duplex MIMO relays after null-space projection and cancellation," in Proc. Asilomar Conf. Signals, Syst. Comput., Nov. 2010, pp. 653-657.

[24] M. Duarte, C. Dick, and A. Sabharwal, "Experiment-driven characterization of full-duplex wireless systems," IEEE Trans. Wireless Commun., vol. 11, no. 12, pp. 4296-4307, Dec. 2012.

[25] M. Jain et al., "Practical, real-time, full duplex wireless," in Proc. ACM MobiCom, Sep. 2011, pp. 301-312.

[26] A. K. Khandani, Shaping the Future of Wireless: Two-Way Connectivity, Jun. 2012. [Online]. Available: http://www.nortel-institute.uwaterloo.ca/ content/ShapingFutureofWirelessTwo-wayConnectivity_18ne2012.pdf

[27] E. Aryafar, M. Khojastepour, K. Sundaresan, S. Rangarajan, and M. Chiang, "MIDU: Enabling MIMO full duplex," in Proc. ACM MobiCom, 2012, pp. 257-268.

[28] M. Duarte et al., "Design and characterization of a full-duplex multiantenna system for WiFi networks," IEEE Trans. Veh. Commun., vol. 63, no. 3, pp. 1160-1177, Mar. 2014.

[29] A. Sahai, G. Patel, and A. Sabharwal, Pushing the Limits of Full-Duplex: Design and Real-Time Implementation, Rice University, Houston, TX, USA, Tech. Rep. TREE1104.

[30] M. E. Knox, "Single antenna full duplex communications using a common carrier," in Proc. IEEE 13th Anпu. WAMICON, 2012, pp. 1-6.

[31] D. Bharadia, E. McMilin, and S. Katti, "Full duplex radios," in Proc. ACM SIGCOMM, 2013, pp. 375-386.

[32] B. Chun, E.-R. Jeong, J. Joung, Y. Oh, and Y. H. Lee, "Pre-nulling for self-interference suppression in full-duplex relays," in Proc. APSIPA $A S C, 2009$, pp. 91-97.

[33] P. Lioliou, M. Viberg, M. Coldrey, and F. Athley, "Self-interference suppression in full-duplex MIMO relays," in Proc. Asilomar Conf. Signals, Syst. Comput., 2010, pp. 658-662.

[34] B. Chun and Y. H. Lee, "A spatial self-interference nullification method for full duplex amplify-and-forward MIMO relays," in Proc. IEEE WCNC, Apr. 2010, pp. 1-6.

[35] D. Senaratne and C. Tellambura, "Beamforming for space division duplexing," in Proc. IEEE ICC, Jun. 2011, pp. 1-5.

[36] T. Riihonen, S. Werner, and R. Wichman, "Mitigation of loopback selfinterference in full-duplex MIMO relays," IEEE Trans. Signal Process., vol. 59, no. 12, pp. 5983-5993, Dec. 2011.

[37] T. Snow, C. Fulton, and W. J. Chappell, "Transmit-receive duplexing using digital beamforming system to cancel self-interference," IEEE Trans. Microw. Theory Tech., vol. 59, no. 12, pp. 3494-3503, Dec. 2011

[38] T. Riihonen et al., "Optimal eigenbeamforming for suppressing selfinterference in full-duplex MIMO relays," in Proc. 45th Аппи. CISS, 2011, pp. 1-6.

[39] E. Everett, "Full-duplex infrastructure nodes: Achieving long range with half-duplex mobiles," M.S. thesis, Rice University, Houston, TX, USA, 2012.

[40] B. Day, A. Margetts, D. Bliss, and P. Schniter, "Full-duplex bidirectional MIMO: Achievable rates under limited dynamic range," IEEE Trans. Signal Process., vol. 60, no. 7, pp. 3702-3713, Jul. 2012.

[41] B. Day, A. Margetts, D. Bliss, and P. Schniter, "Full-duplex MIMO relaying: Achievable rates under limited dynamic range," IEEE J. Sel. Areas Commun., vol. 30, no. 8, pp. 1541-1553, Sep. 2012.
[42] F. O'Hara and G. Moore, "A high performance CW receiver using feedthru nulling," Microw. J., vol. 6, pp. 63-71, Sep. 1963.

[43] A. G. Stove, "Linear FMCW radar techniques," Proc. Inst. Elect. Eng. F (Radar Signal Process.), vol. 139, no. 5, pp. 343-350, Oct. 1992.

[44] H. Suzuki, K. Itoh, Y. Ebine, and M. Sato, "A booster configuration with adaptive reduction of transmitter-receiver antenna coupling for pager systems," in Proc. IEEE Veh. Technol. Conf._Fall, 1999, vol. 3, pp. 1516-1520.

[45] K. Lin, Y. E. Wang, C.-K. Pao, and Y.-C. Shih, "A Ka-Band FMCW radar front-end with adaptive leakage cancellation," IEEE Trans. Microw. Theory Tech., vol. 54, no. 12, pp. 4041-4048, Dec. 2006.

[46] J.-W. Jung et al., "TX leakage Cancellation via a micro controller and high TX-to-RX isolations covering an UHF RFID frequency band of 908 to $914 \mathrm{MHz}, "$ IEEE Microw. Wireless Compon. Lett., vol. 18, no. 10, pp. $710-712$, Oct. 2008

[47] G. Lasser, R. Langwieser, and A. L. Scholtz, "Broadband suppression properties of active leaking carrier cancellers," in Proc. IEEE Int. Conf. RFID, 2009, pp. 208-212.

[48] P. Pursula, M. Kiviranta, and H. Seppa, "UHF RFID reader with reflected power canceller," IEEE Microw. Wireless Compon. Lett., vol. 19, no. 1, pp. 48-50, Jan. 2009.

[49] H. Hamazumi, K. Imamura, N. Iai, K. Shibuya, and M. Sasaki, "A study of a loop interference canceller for the relay stations in an SFN for digital terrestrial broadcasting," in Proc. IEEE Global Telecommun. Conf., 2000, vol. 1, pp. 167-171.

[50] K. Lin, R. Messerian, and Y. Wang, "A digital leakage cancellation scheme for monostatic FMCW radar," in Proc. IEEE MTT-S Int. Microw. Symp. Dig., 2004, vol. 2, pp. 747-750.

[51] J. Ma, G. Li, J. Zhang, T. Kuze, and H. Iura, "A new coupling channel estimator for cross-talk cancellation at wireless relay stations," in Proc. IEEE Global Telecommun. Conf., 2009, pp. 1-6.

[52] E. A. Rodriguez, R. L. Valcarce, T. Riihonen, S. Werner, and R. Wichman, "Adaptive self-interference cancellation in wideband fullduplex decode-and-forward MIMO relays," in Proc. IEEE Workshop SPAWC, Jun. 2013, pp. 370-374.

[53] E. A. Rodriguez, R. L. Valcarce, T. Riihonen, S. Werner, and R. Wichman, "Autocorrelation-based adaptation rule for feedback equalization in wideband full-duplex amplify-and- forward MIMO relays," in Proc. IEEE ICASSP, May 2013, pp. 4968-4972.

[54] T. Riihonen, S. Werner, R. Wichman, and E. Zacarias, "On the feasibility of full-duplex relaying in the presence of loop interference," in Proc. IEEE Workshop Signal Process. Adv. Wireless Commun., 2009, pp. 275-279.

[55] H. Ju, E. Oh, and D. Hong, "Catching resource-devouring worms in nextgeneration wireless relay systems: Two-way relay and full-duplex relay," IEEE Commun. Mag., vol. 47, no. 9, pp. 58-65, Sep. 2009.

[56] D. Ng, E. Lo, and R. Schober, "Dynamic resource allocation in MIMOOFDMA systems with full-duplex and hybrid relaying," IEEE Trans. Commun., vol. 60, no. 5, pp. 1291-1304, May 2012.

[57] T. Riihonen, S. Werner, and R. Wichman, "Hybrid full-duplex/halfduplex relaying with transmit power adaptation," IEEE Trans. Wireless Commun., vol. 10, no. 9, pp. 3074-3085, Sep. 2011.

[58] A. K. Khandani, "Methods for spatial multiplexing of wireless two-way channels," U.S. Patent 7817641 , Oct. 2010.

[59] D. Guo and L. Zhang, "Virtual full-duplex wireless communications via rapid on-off-division duplex," in Proc. Allerton Conf. Commun., Control Comput., 2010, pp. 412-419.

[60] L. Zhang, J. Luo, and D. Guo, "Neighbour discovery for wireless networks via compressed sensing," Performance Eval., vol. 70, pp. 457471,2013

[61] L. Zhang and D. Guo, "Virtual full-duplex wireless broadcasting via compressed sensing," IEEE/ACM Trans. Netw., to be published.

[62] M. Steer, Microwave and RF Engineering: A Systems Approach. Raleigh, NC, USA: Scitech Publishing Inc., 2010.

[63] S. M. Wentworth, Applied Electromagnetics: Early Transmission Lines Approach. Hoboken, NJ, USA: Wiley, 2007.

[64] P. Beasley and A. Stove, "Pilot-An example of advanced FMCW techniques," Proc. IEE Colloq. High Time-Bandwidth Product Waveforms Radar Sonar, pp. 10/1-10/5, 1991.

[65] EPC Radio Frequency Identity Protocols, Class-l Generation-2 UHF RFID Protocol for Communications at $860 \mathrm{MHz}$ to $960 \mathrm{MHz}$, ISO180006C, 2008.

[66] R. Isberg and W. Lee, "Performance tests of a low power cellular enhancer in a parking garage," in Proc. IEEE 39th Veh. Technol. Conf., 1989 , pp. 542-546.

[67] W. T. Slingsby and J. P. McGeehan, "A high-gain cell enhancer," in Proc. IEEE 42nd Veh. Technol. Conf., 1992, vol. 2, pp. 756-758. 
[68] W. Slingsby and J. McGeehan, "Antenna isolation measurements for onfrequency radio repeaters," in Proc. 9th Int. Conf. (Conf. Publ. No. 407) Antennas Propag., Apr. 1995, vol. 1, pp. 239-243.

[69] S. J. Kim et al., "Adaptive feedback interference cancellation system (AF-ICS)," in Proc. IEEE MTT-S Int. Microw. Symp. Dig., 2003, vol. 1, pp. 627-630.

[70] T. Riihonen, R. Wichman, and J. Hamalainen, "Co-phasing full-duplex relay link with non-ideal feedback information," in Proc. IEEE Int. Symp. Wireless Commun. Syst., Oct. 2008, pp. 263-267.

[71] T. Riihonen, S. Werner, J. Cousseau, and R. Wichman, "Design of cophasing allpass filters for full-duplex OFDM relays," in Proc. Asilomar Conf. Signals, Syst. Comput., Oct. 2008, pp. 1030-1034.

[72] H. Ju, E. Oh, and D. Hong, "Improving efficiency resource usage in twohop full duplex relay systems based on resource sharing and interference cancellation," IEEE Trans. Wireless Commun., vol. 8, no. 8, pp. 39333938, Aug. 2009.

[73] Physical Layer for Relaying Operation (Release 10), LTE spec, 3GPP TS 36.216, June 2011.

[74] O. Somekh, O. Simeone, H. Poor, and S. Shamai, "Cellular systems with full-duplex amplify-and-forward relaying and cooperative basestations," in Proc. IEEE Int. Symp. Inf. Theory, 2007, pp. 16-20.

[75] V. Cadambe and S. Jafar, "Can feedback, cooperation, relays and full duplex operation increase the degrees of freedom of wireless networks?" in Proc. IEEE ISIT, Jul. 2008, pp. 1263-1267.

[76] S. Simoens, O. Munoz-Medina, J. Vidal, and A. del Coso, "On the Gaussian MIMO relay channel with full channel state information," IEEE Trans. Signal Process., vol. 57, no. 9, pp. 3588-3599, Sep. 2009.

[77] S. Sohaib and D. K. C. So, "Asynchronous polarized cooperative MIMO communication," in Proc. Veh. Technol. Conf., Apr. 2009, pp. 1-5.

[78] T. Riihonen, K. Haneda, S. Werner, and R. Wichman, "SINR analysis of full-duplex OFDM repeaters," in Proc. PIMRC, Sep. 2009, pp. 3169-3173.

[79] K. Yamamoto, K. Haneda, H. Murata, and S. Yoshida, "Optimal transmission scheduling for a hybrid of full- and half-duplex relaying," IEEE Commun. Lett., vol. 15, no. 3, pp. 305-307, Mar. 2011.

[80] Y. K. Song and D. Kim, "Convergence of distributed power control with full-duplex amplify-and-forward relays," in Proc. Int. Conf. Wireless Commun. Signal Process., 2009, pp. 1-5.

[81] Y. Y. Kang and J. H. Cho, "Capacity of MIMO wireless channel with full-duplex amplify-and-forward relay," in Proc. IEEE 20th Int. Symp. Pers., Indoor Mobile Radio Commun., 2009, pp. 117-121.

[82] T. Baranwal, D. Michalopoulos, and R. Schober, "Outage analysis of multihop full duplex relaying," IEEE Commun. Lett., vol. 17, no. 1, pp. 63-66, Jan. 2013.

[83] D. W. Bliss and S. Govindasamy, Adaptive Wireless Communications. MIMO Channels and Networks. Cambridge, U.K.: Cambridge Univ. Press, 2013.

[84] B. Radunovic et al., "Rethinking Indoor Wireless: Low Power, Low Frequency, Full Duplex," Microsoft Res., Redmond, WA, USA, Tech. Rep., 2009.

[85] M. Duarte, "Full-Duplex Wireless: Design, Implementation and Characterization," Ph.D. dissertation, Rice University, Houston, TX, USA, Apr., 2012.

[86] L. W. Fullerton, "Full Duplex Ultrawide-Band Communication System and Method," U.S. Patent 5687 169, Nov. 11, 1997.

[87] S. N. Stitzer, "Full Duplex Communication System Apparatus Using Frequency Selective Limiters," U.S. Patent 4325 140, Apr. 13, 1982.

[88] A. Sahai, G. Patel, C. Dick, and A. Sabharwal, "Understanding the impact of phase noise on active cancellation in wireless full-duplex," in Proc. Asilomar Conf. Signals, Syst. Comput., 2012, pp. 29-33.

[89] A. Sahai, G. Patel, C. Dick, and A. Sabharwal, "On the impact of phase noise on active cancellation in wireless full-duplex," IEEE Trans. Veh. Technol., vol. 62, no. 9, pp. 4494-4510, Nov. 2013.

[90] D. W. Bliss, T. M. Hancock, and P. Schniter, "Hardware phenomenological effects on cochannel full-duplex MIMO relay performance," in Proc. IEEE Asilomar Conf. Signals, Syst. Comput., 2012, pp. 34-39.

[91] E. Ahmed, A. Eltawil, and A. Sabharwal, "Rate gain region and design tradeoffs for full-duplex wireless communications," IEEE Trans. Wireless Commun., vol. 12, no. 7, pp. 3556-3565, Jul. 2013.

[92] V. Syrjala, M. Valkama, L. Anttila, T. Riihonen, and D. Korpi, "Analysis of oscillator phase-noise effects on self-interference cancellation in dull-duplex OFDM radio transceivers," IEEE Trans. Wireless Commun., vol. 13, no. 6, pp. 2977-2990, Jun. 2014.

[93] D. Korpi et al., "Full-duplex transceiver system calculations: Analysis of ADC and linearity challenges," IEEE Trans. Wireless Commun., to be published.
[94] D. Korpi et al., "Advanced self-interference cancellation and multiantenna solutions for full-duplex radios," in Proc. Asilomar Conf. Signals, Syst. Comput., 2013.

[95] L. Anttila, D. Korpi, and V. Syrjälä, "Cancellation of power amplifier induced nonlinear self-interference in full-duplex transceivers," in Proc. 47th Asilomar Conf. Signals, Syst. Comput., 2013, pp. 1-6.

[96] Y.-S. Choi and H. Shirani-Mehr, "Simultaneous transmission and reception: Algorithm, design and system level performance," Wireless Commun., vol. 12, no. 12, pp. 5992-6010, Dec. 2013.

[97] H. Holma and A. Toskala, Eds., WCDMA for UMTS: Radio Access for Third Generation Mobile Communications, 3rd ed. Hoboken, NJ, USA: Wiley, 2004.

[98] A. Kiayani, L. Anttila, and M. Valkama, "Digital suppression of power amplifier spurious emissions at receiver band in FDD transceivers," IEEE Signal Process. Lett., vol. 21, no. 1, pp. 69-73, Jan. 2014.

[99] M. Omer, R. Rimini, P. Heidmann, and J. Kenney, "A compensation scheme to allow full duplex operation in the presence of highly nonlinear microwave components for 4G systems," in Proc. IEEE MTT-S Int. MTT, Jun. 2011, pp. 1-4.

[100] A. Frotzscher and G. Fettweis, "Digital compensation of transmitter leakage in FDD zero-IF receivers," Trans. Emerg. Telecommun. Technol., vol. 23, no. 2, pp. 105-120, Mar. 2012.

[101] A. Kiayani, L. Anttila, and M. Valkama, "Modeling and dynamic cancellation of TX-RX leakage in FDD transceivers," in Proc. IEEE 56th Int. MWSCAS, Aug. 2013, pp. 1089-1094.

[102] C. S. Vaze and M. K. Varanasi, The Degrees of Freedom of MIMO Networks With Full-Duplex Receiver Cooperation But No CSIT 2012, arXiv preprint arXiv:1209.1291

[103] Q. Geng, S. Kannan, and P. Viswanath, Interactive Interference Alignment 2012, arXiv preprint arXiv:1211.0985.

[104] A. Sahai, S. Diggavi, and A. Sabharwal, "On degrees of freedom of fullduplex uplink/downlink channel," in Proc. IEEE ITW, Sep. 2013, pp. 1-5.

[105] J. Bai and A. Sabharwal, "Decode-and-cancel for interference cancellation in a three-node full-duplex network," in Proc. Asilomar Conf. Signals, Syst. Comput., 2012, pp. 1285-1289.

[106] J. Bai and A. Sabharwal, "Distributed full-duplex via wireless sidechannels: Bounds and protocols," IEEE Trans. Wireless Commun., vol. 12, no. 8, pp. 4162-4173, Aug. 2013.

[107] W. Cheng, X. Zhang, and H. Zhang, Full duplex wireless communications for cognitive radio networks 2011, arXiv:1105.0034v1.

[108] W. Schacherbauer, A. Springer, T. Ostertag, C. Ruppel, and R. Weigel, "A flexible multiband frontend for software radios using high IF and active interference cancellation," in Proc. IEEE Int. Microw. Symp., 2001, pp. 1085-1088.

[109] A. Raghavan, E. Gebara, E. M. Tentzeris, and J. Laskar, "Analysis and design of an interference canceller for collocated radios," IEEE Trans. Microw. Theory Tech., vol. 53, no. 11, pp. 3498-3508, Nov. 2005.

[110] N. Singh et al., "Efficient and fair MAC for wireless networks with self-interference cancellation," in Proc. Int. Symp. WiOpt, May 2011, pp. 94-101.

[111] S. Sen, R. Roy Choudhury, and S. Nelakuditi, "No time to countdown: Migrating backoff to the frequency domain," in Proc. ACM MobiCom, New York, NY, USA, 2011, pp. 241-252.

[112] P. Weeraddana, M. Codreanu, M. Latva-aho, and A. Ephremides, "On the effect of self-interference cancelation in multihop wireless networks," EURASIP J. Wireless Commun. Netw., vol. 2010, no. 1, p. 513952, 2010.

[113] D. Ramirez and B. Aazhang, "Optimal routing and power allocation for wireless networks with imperfect full-duplex nodes," IEEE Trans. Wireless Commun., vol. 12, no. 9, pp. 4692-4704, Sep. 2013.

[114] X. Fang, D. Yang, and G. Xue, "Distributed algorithms for multipath routing in full-duplex wireless networks," in Proc. IEEE 8th Int. Conf. MASS, Washington, DC, USA, 2011, pp. 102-111.

[115] W. Mesbah and T. Davidson, "Optimal power allocation for full-duplex cooperative multiple access," in Proc. IEEE ICASSP, 2006, vol. 4 , p. IV.

[116] M. Gan, D. Guo, and X. Dai, Distributed Ranging and Localization for Wireless Networks via Compressed Sensing 2013, arXiv preprint arXiv: 1308.3548

[117] D. N. C. Tse and P. Viswanath, Fundamentals of Wireless Communication. Cambridge, U.K.: Cambridge Univ. Press, 2005.

[118] E. Magistretti, K. K. Chintalapudi, B. Radunovic, and R. Ramjee, "WiFiNano: Reclaiming WiFi efficiency through 800 ns slots," in Proc. ACM MobiCom, 2011, pp. 37-48.

[119] P. Pursula and H. Seppa, "Hybrid transformer-based adaptive RF front end for UHF RFID mobile phone readers," in Proc. IEEE Int. Conf. RFID, 2008, pp. 150-155. 
[120] T. Riihonen and R. Wichman, "Analog and digital self-interference cancellation in full-duplex MIMO-OFDM transceivers with limited resolution in A/D conversion," in Proc. Asilomar Conf. Signals, Syst. Comput., November 2012, pp. 45-49.

[121] T. Schenk, RF Imperfections in High-Rate Wireless Systems: Impact and Digital Compensation. Dordrecht, The Netherlands: Springer-Verlag, 2008.

[122] E. Ahmed, A. M. Eltawil, and A. Sabharwal, "Self-interference cancellation with nonlinear distortion suppression for full-duplex systems," CoRR, vol. abs/1307.3796, 2013.

[123] E. Antonio-Rodriguez, R. Lopez-Valcarce, T. Riihonen, S. Werner, and R. Wichman, "SINR optimization in wideband full-duplex MIMO relays under limited dynamic range," presented at the IEEE Sensor Array Multichannel Signal Processing Workshop, Coruña, Spain, Jun. 2014

[124] T. Riihonen, P. Mathecken, and R. Wichman, "Effect of oscillator phase noise and processing delay in full-duplex OFDM repeaters," in Proc. Asilomar Conf. Signals, Syst. Comput., Nov. 2012, pp. 1947-1951.

[125] E. Everett, D. Dash, C. Dick, and A. Sabharwal, "Self-interference cancellation in multi-hop full-duplex networks via structured signaling," in Proc. Allerton Conf. Commun., Control, Comput., Nov. 2011, pp. $1619-1626$.

[126] V. Aggarwal, M. Duarte, A. Sabharwal, and N. K. Shankaranarayanan, "Full- or half-duplex? A capacity analysis with bounded radio resources," in Proc. Inf. Theory Workshop, 2012, pp. 207-211.

[127] S. Barghi, A. Khojastepour, K. Sundaresan, and S. Rangarajan, "Characterizing the throughput gain of single cell MIMO wireless systems with full duplex radios," in Proc. Int. Symp. WiOpt, May 2012, pp. 68-74.

[128] F. Baccelli and B. Błaszczyszyn, Stochastic Geometry and Wireless Networks: Volume I Theory and Volume II Applications, vol. 4, Foundations and Trends in Networking. Delft, The Netherlands: NoW Publishers, 2009.

[129] M. Haenggi, Stochastic Geometry for Wireless Networks. Cambridge, U.K.: Cambridge Univ. Press, 2012.

[130] C. Fragouli, J.-Y. L. Boudec, and J. Widmer, "Network coding: An instant primer," ACM SIGCOMM Comput. Commun. Rev., vol. 36, no. 1, pp. 63-68, Jan. 2006.

[131] S. Zhang, S. C. Liew, and P. P. Lam, "Physical-layer network coding," in Proc. ACM MobiCom, 2006, pp. 358-365.

[132] S. Katti et al., "XORs in the air: Practical wireless network coding," IEEE/ACM Trans. Netw., vol. 16, no. 3, pp. 497-510, Jun. 2008.

[133] Y. Polyanskiy, H. V. Poor, and S. Verdú, "Channel coding rate in the finite blocklength regime," IEEE Trans. Inf. Theory, vol. 56, no. 5, pp. 23072359, May 2010.

[134] T.-Y. Chen, A. R. Williamson, and R. D. Wesel, "Variable-length coding with feedback: Finite-length codewords and periodic decoding," in Proc. IEEE Int. Symp. Inf. Theory, 2013, pp. 2547-2551.

[135] S. Verdú, Multiuser Detection. Cambridge, U.K.: Univ. Press, 1998.

[136] M. L. Honig, Advances in Multiuser Detection. Hoboken, NJ, USA: Wiley, 2009.

[137] S. H. Han and J. H. Lee, "An overview of peak-to-average power ratio reduction techniques for multicarrier transmission," IEEE Wireless Commun., vol. 12, no. 2, pp. 56-65, Apr. 2005.

[138] "Comparison of PAR and Cubic Metric for Power De-Rating," SophiaAntipolis, France, Tech. Rep. R1-040642, May 2004, Motorola.

[139] R. N. Braithwaite, "The effects of power amplifier nonlinearities and CFR on 64QAM HSDPA waveforms," in Proc. Microw. Meas. Conf. (ARFTG), 2013, pp. 1-3.

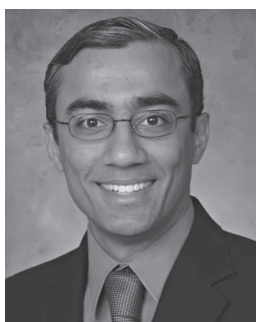

Ashutosh Sabharwal (S'91-M'99-SM'04-F'14) received the $\mathrm{B}$.Tech. degree from the Indian Institute of Technology, New Delhi, India, in 1993 and the M.S. and Ph.D. degrees from The Ohio State University, Columbus, OH, USA, in 1995 and 1999, respectively. He is currently a Professor with the Department of Electrical and Computer Engineering, Rice University, Houston, TX, USA. His research interests include information theory and communication algorithms for wireless systems. Dr. Sabharwal was a recipient of the Presidential Dissertation Fellowship Award in 1998 and is the Founder of the Wireless Open-Access Research Platform Project (http://warp.rice.edu).

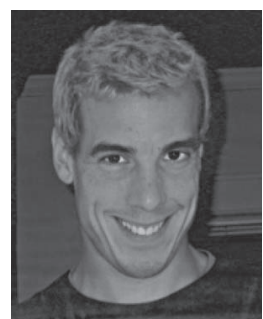

Philip Schniter (F'14) received the B.S. and M.S. degrees in electrical engineering from the University of Illinois at Urbana-Champaign, IL, USA, in 1992 and 1993, respectively, and the Ph.D. degree in electrical engineering from Cornell University, Ithaca, NY, USA, in 2000. From 1993 to 1996, he was with Tektronix Inc., Beaverton, OR, USA, as a Systems Engineer. After receiving the Ph.D. degree, he joined the Department of Electrical and Computer Engineering, The Ohio State University, Columbus, OH, USA, where he is currently a Professor. In 2008-2009, he was a Visiting Professor at Eurecom, Sophia Antipolis, France, and Supelec, Gif-sur-Yvette, France. His areas of interest currently include signal processing, machine learning, and wireless communications and networks. Dr. Schniter was a recipient of the National Science Foundation CAREER Award in 2003.

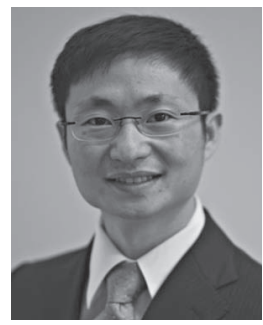

Dongning Guo (S'98-M'05-SM'11) received the B.Eng. degree from the University of Science and Technology of China, Hefei, China, the M.Eng. degree from the National University of Singapore, Singapore, and the M.A. and Ph.D. degrees from Princeton University, Princeton, NJ, USA. He joined the faculty of Northwestern University, Evanston, IL, USA, in 2004, where he is currently an Associate Professor in the Department of Electrical Engineering and Computer Science. He was an R\&D Engineer in the Center for Wireless Communications, Singapore, from 1998 to 1999. He has been an Associate Editor of the IEEE TRANSACTIONS ON INFORMATION THEORY and is an Editor of Foundations and Trends in Communications and Information Theory. He was a recipient of the Huber and Suhner Best Student Paper Award at the International Zurich Seminar on Broadband Communications in 2000 and was a corecipient of the IEEE Marconi Prize Paper Award in Wireless Communications in 2010. He was also a recipient of the NSF CAREER Award in 2007.

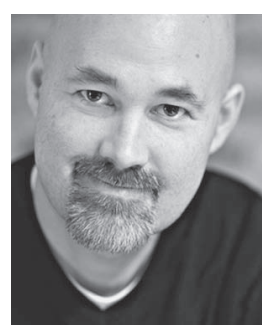

Daniel W. Bliss (SM'10) received the B.S.E.E. degree in electrical engineering from Arizona State University (ASU), Phoenix, AZ, USA, in 1989 and the M.S. and Ph.D. degrees in physics from the University of California, San Diego, La Jolla, CA, USA, in 1995 and 1997, respectively. His doctoral work (1993-1997) was in the area of high-energy particle physics. He is an Associate Professor with the School of Electrical, Computer and Energy Engineering, ASU. His current research topics include multiple-input-multiple-output (MIMO) wireless communications, MIMO radar, cognitive radios, radio network performance, geolocation, and statistical signal processing for anticipatory physiological analytics. He has been the Principal Investigator on numerous programs with applications to radio, radar, and medical monitoring. He has made significant contributions to robust multiple-antenna communications, including theory, patents, and the development of advanced prototypes. He is responsible for some of the foundational MIMO radar literature.

Before moving to ASU, he was a Senior Member of Technical Staff at MIT Lincoln Laboratory (1997-2012). Employed by General Dynamics (1989-1993), he designed rocket avionics and performed magnetic field calculations and optimization for high-energy particle-accelerator superconducting magnets. 


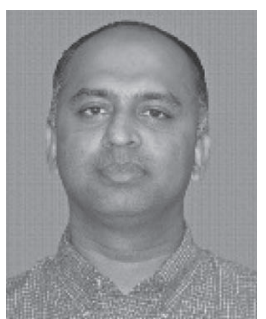

Sampath Rangarajan received the M.S. degree in electrical and computer engineering and the Ph.D. degree in computer science from the University of Texas at Austin, TX, USA. He heads the Mobile Communications and Networking Research Department, NEC Laboratories America, Inc., Princeton, NJ, USA. Previously, he was with the Networking Research Center, Bell Laboratories, Holmdel, NJ. Prior to that, he was a Cofounder and Vice President of Technology at Ranch Networks, a venture funded startup in the IP networking space. Earlier, he was a Researcher with the Systems and Software Research Center, Bell Laboratories, Murray Hill, NJ. Before joining Bell Laboratories, he was an Assistant Professor with the Department of Electrical and Computer Engineering, Northeastern University, Boston, MA, USA. His research interests span the areas of mobile communications, mobile networks, and distributed systems. Dr. Rangarajan has been on the Editorial Boards of the IEEE TRANSACTIONS ON COMPUTERS, IEEE TRAnsactions on PARALlel AND Distributed Systems, and ACM Mobile Computing and Communications Review.

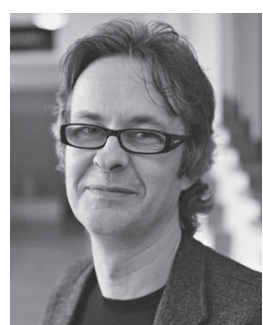

Risto Wichman received the M.Sc. and D.Sc. (Tech.) degrees in digital signal processing from Tampere University of Technology, Tampere, Finland, in 1990 and 1995, respectively. From 1995 to 2001, he worked at Nokia Research Center as a Senior Research Engineer. In 2002, he joined the Department of Signal Processing and Acoustics, School of Electrical Engineering, Aalto University, Espoo, Finland, where he has been a Full Professor since 2008. 